\title{
Efficient DWT-based SVD Watermarked 3D Video Transmission over MC-CDMA Wireless Channel
}

\author{
Walid El-Shafai \\ Menoufia University \\ s El-Rabaie \\ Menoufia University \\ o Zahran \\ Menoufia University \\ M El-Halawany \\ Menoufia University \\ F E. Abd El-Samie \\ Menoufia University
}

Eman Mohamed Elbakary ( $\nabla$ eman_elbakary449@yahoo.com )

Menoufia University https://orcid.org/0000-0001-5101-9981

\section{Research Article}

Keywords: 3D Video, Chaotic interleaving, Convolution codes, DWT, SVD, Linear equalization, MC-CDMA, Wireless channel.

Posted Date: March 15th, 2021

DOl: https://doi.org/10.21203/rs.3.rs-220289/v1

License: (c) (i) This work is licensed under a Creative Commons Attribution 4.0 International License.

Read Full License 


\title{
Efficient DWT-based SVD Watermarked 3D Video Transmission over MC-CDMA Wireless Channel
}

\author{
E. M. El-Bakary, Walid El-Shafai, S. El-Rabaie, O. Zahran, M. El-Halawany, F. E. Abd El-Samie \\ Department of Electronics and Electrical Communications Engineering, Faculty of Electronic \\ Engineering, Menoufia University, 32952 Menouf, Egypt.
}

\begin{abstract}
Three-Dimensional Video (3DV) communication through wireless channels suffers from bit-streams losses. Therefore, the efficient performance of 3DV transmission techniques over wireless networks is a considerable hot research topic. A high compression ratio must be introduced to meet future bandwidth restriction for optimized 3DV transmission. Unfortunately, the compressed 3DV bitstreams are more sensitive and vulnerable to packet losses. In this paper, we propose the application of chaotic Baker interleaving with equalization and convolution coding for efficient Discrete Wavelet Transform (DWT)-based Singular Value Decomposition (SVD) watermarked 3DV transmission over a Multi-Carrier Code Division Multiple Accesses (MC-CDMA) wireless channel. Firstly, the compressed 3DV frames are watermarking using the DWT+SVD watermarking process. After that, chaotic interleaving is applied to minimize the channel effects on the transported bit-streams and it also adds an encryption to the transported 3DV frames. To test the performance of the proposed hybrid techniques; several experiments on different (DWT+SVD) watermarked 3DV frames have been tested. The experimental results confirm that the received watermarked 3DV frames still have high Peak Signal-to-Noise Ratios (PSNRs) and efficient watermark extraction is possible.
\end{abstract}

Keywords 3D Video, Chaotic interleaving, Convolution codes, DWT, SVD, Linear equalization, MC-CDMA, Wireless channel.

\section{Introduction}

The 3DV has expected to quickly replace traditional 2D video in different applications [1-3]. In 3DV system, the original 3DV data is a collection of video streams taken for the same object by multiple cameras, simultaneously. The 3DV transmission over wireless networks has increased dramatically. Thus, in order to transmit 3DV over limited bandwidth wireless networks; a highly efficient compression algorithm must be applied while maintaining a high reception quality. The highly compressed 3DV transmission over wireless networks is always subject to random and burst errors [45]. There is a variety of techniques that can be utilized to limit the impact of packet losses incurred in the transmission process [6-7]. In the literature [8-9], the Forward Error Correction (FEC) and the Automatic Repeated Request (ARQ) techniques have been introduced. Unfortunately, they raise the transmission bit rate and present some delay. It is impossible to retransmit all corrupted or lost MacroBlocks (MBs) due to the delay constraints on real-time video communication. Therefore, there is a need for robust signal processing schemes for efficient 3DV transmission without increasing the delay or using more bandwidth resources.

For efficient 3DV transmission, the 3DV system must exploit the spatio-temporal correlations between frames within each video as well as the inter-view correlations between the video streams to 
increase the compression efficiency. On the other hand, extremely compressed video bit streams are brittle to the transmission errors. The 3DV streaming through wireless channels is always subject to burst and random corruptions [4]. Due to the predictive coding structure of the 3DV system, which utilizes the intra (I-frames) and inter- (P and B-frames) coded frames, errors could propagate to the subsequent frames and to the adjacent views and result in poor visual quality [5]. In real-world video communication applications, the compression and robust transmission are important objectives for reliable video transmission. Therefore, the mandatory objective is to transmit as minimum amount of information as possible with the highest degree of security. To attain both objectives, we need to use both watermarking and compression together. Also, because the 3DV transmission through wireless channels faces severe conditions such as burst errors, there is a need to minimize the effect of these errors. We use the Peak Signal-to-Noise Ratio (PSNR) to measure the efficiency of the received 3DV frames.

The watermarking is an attractive technique that are applied for reliable multimedia communication. It is used in a wide range of applications, and it has become an interesting scheme, especially when it is used in conjunction with cryptography. The goal of watermarking algorithms is achieving detectability, robustness, and high capacity of the hidden data. The watermarking process embeds the watermarks into the transmitted data in an invisible manner. It inserts the secret data to be hidden in the multimedia transmitted signal to achieve authentication. The number of hidden bits represents the payload which determines the invisibility requirement. The 3DV contains a number of different frames, the watermark is embedded into each these frames using the Discrete Wavelet Transform (DWT)-based Singular Value Decomposition (SVD) watermarking technique. Each 3D video frame component of the Y CB CR system is divided into non-overlapping blocks with size of $16 \times 16$ pixels, and then the watermark is embedded into these blocks using the SVD+DWT watermarking techniques.

The 3DV transmission through wireless channels faces severe conditions such as burst errors, thus there is a need to minimize the effect of these errors. In this paper, we apply an efficient interleaving scheme on the transported binary data extracted from the (DWT+SVD) watermarked 3DV frames at the transmitter. Moreover, we employ convolution coding and equalization. The proposed convolution code is based on the Viterbi algorithm. The proposed convolution coder presents redundant bits into the stream of the transmitted data through the use of linear shift registers. The introduced redundant bits are used to detect and correct the accumulated errors in the received data bit streams at the receiver. Chaotic interleaving is based on Baker map is employed [10-11]. One of the most attractive merits of the chaotic map interleaving is reducing the channel effects. Another advantage is achieving a degree of encryption to the transmitted data. At the receiving part, the linear equalization is employed for eliminating the effects of the Inter-Symbol Interference (ISI) and Multiple-Access Interference (MAI) [12-13].

The organization of this paper is as follows. Section 2 introduces some of existing hybrid watermarking related works. Section 3 presents the 3DV prediction structure (3DV-PS). Section 4 presents the MC-CDMA system model which is used as a transmission platform for the compressed (DWT+SVD) watermarked 3DV frames. Section 5 discusses a joint framework comprising (DWT+SVD) watermarking, chaotic Baker map interleaving, convolution coding, and linear 
equalization for reliable 3DV transmission. Section 6 presents the simulation results and comparative analysis. Finally, section 7 summarizes the concluding remarks.

\section{Related Work}

The 3DV comprises diverse video streams captured by different cameras around an object. Therefore, it is an imperative assignment to fulfil efficient compression to transmit and store the 3DV content in a sufficient compressed form to attain future resource bounds whilst preserving a decisive reception quality. Also, the security of the transmitted 3DV is a critical issue for protecting its content copyright. Due to the fast progress in network development, humans can easily and arbitrarily distribute or access digital multimedia data from networks. The ownership security has become an important issue for individuals, and it requires more interest. Thus, there is a significant threat to copyright owners and digital multimedia producers to conserve their multimedia from intruder prospection to avert loss in transmitted data [1]. The watermarking is one of the most favourable methods to secure digital multimedia files in the domains of copyright protection and data authentication, where a watermark secret code is inserted in the transmitted digital multimedia, and it contains the information about the creator of the media, the copyright owner, or the authorized user. The utilization of digital watermarks for efficient video transmission can be beneficial to ensure copyright. A digital watermark can be embedded either in a compressed video or uncompressed video [2]. Video information are always transported and stored in the form of compressed data. The uncompressed video watermarking techniques can also be utilized for the compressed-video bit streams. However, they require complete video re-encoding and decoding for the watermark insertion or extraction. In different cases, the complete video stream decoding process is not recommended. So, the compressed video watermarking has recently acquired more attentiveness. Furthermore, the watermark insertion and extraction in compressed data has less computations, because the complete reencoding and decoding of the transmitted stream is not required for embedding and extraction of the watermark bits.

Video compression is the process of reducing the size of the transmitted video data, thereby, reducing the bandwidth required for the digital representation of the video signals. Video signals can be compressed because of the spatial, spectral, and temporal correlation inherent in these signals. Spatial correlation is the correlation between neighboring samples in each video frame. Temporal refers to correlation between samples in different frames but at the same pixel position. Spectral correlation is the correlation between samples of the same source from multiple sensors. Compression technology can result in reduction in transmission time due to less data being transmitted. It also decreases the storage requirements as there is less data. However, signal quality, implementation complexity, and the introduction of communication delay are potential negative factors that should be considered when choosing the compression technology. Therefore, it is known that compressed video frames transmitted over wireless media are not restored efficiently as some information is usually lost during the compression process in addition to the increased computational complexity and time.

There are several compression techniques which were developed for efficient compression of the video data. The mainly used techniques are the DCT compression and DWT compression. In video 
compression, the video is divided into individual frames, and then various techniques of compression are applied on each frame. The DCT is a lossy compression technique, where an $N \times N$ image block is transformed from the spatial domain to the DCT domain. The DCT decomposes the signal into spatial frequency components called DCT coefficients. In the DCT compression technique, the video is initially converted into various frames. Each frame is divided again into small blocks, and then the DCT is applied to each frame. After applying the DCT, each pixel value is converted into the DCT domain. This conversion process is takes place in such a way that the low frequencies are on the topleft and the higher frequencies are on the bottom right of the DCT block. Then, the quantization is performed resulting in integer DCT coefficients through scaling. By applying Inverse Discrete Cosine Transform (IDCT), compressed frames are obtained. With the DWT, the compression is directly applied on the frame as a whole (i.e., there is no need to divide the frame into smaller blocks). The main intention of this compression technique is storing the frame data with as smaller space as possible. The compression process with the SPIHT technique can be also used to minimize the size of original image or video frame to be transmitted over a communication channel. The objective of using the SPIHT technique is to decrease the required bandwidth through decreasing the amount of data transmitted [11].

Recently, several video encoding standards have emerged. The objective of an encoding standard is to achieve high data compression, while maintaining an acceptable quality. The 3D-MVC and 3DHEVC are efficient and they are the most recent encoding standards used in different applications. The 3D-HEVC has received a broad attentiveness, and it is expected to rapidly take place of traditional 2D video coding in numerous applications [3]. The predictive 3D H.265/HEVC framework is used to compress the transmitted 3DV sequences. Thus, to transport the 3DV over limited-resources networks, a highly efficient compression standard must be applied, whilst preserving a high reception quality.

With the emerging evolution of 3DV applications, the security and copyright protection have become important aspects of the 3DV content storage and transmission. Multimedia watermarking techniques are employed for protecting the 3DV data copyright. Multimedia watermarking methods are classified into two main categories; spatial- and transform-domain methods. The spatial-domain methods hide the watermark in the given video frames by directly adjusting their pixel values. They are simple to carry out and need less computations. Unfortunately, they are not robust enough to attacks. The transform-domain watermarking methods adjust the video frames coefficients in a certain transform domain based on the adopted watermark embedding method. The transform-domain watermarking methods achieve more robustness than those of the spatial-domain methods. Therefore, one of the main contributions of this paper is to present robust and reliable compressed-video watermarking techniques for efficient transmission of 3DV compressed bit streams. These techniques have the following characteristics:

1. Quality. The quality of the watermarked 3DV frames resulting from the embedding process is maintained as high as possible by efficiently choosing the most suitable domain for watermark embedding.

2. Robustness and Security. The proposed watermark embedding and extraction procedures are robust. 
There are few research works on 3DV data watermarking, and most of them deal with Depth-Based Image Rendering (DBIR). Thus, 3DV watermarking is still in its rudimentary phase. A watermarking method in the wavelet-domain for stereo images was introduced in [14]. It depends on extracting the depth map from the stereo pairs for watermark embedding. In [15], a visual model method for watermarking of High Definition (HD) stereo-images in DCT domain was presented. It is based on the visual sensitivity of the human eye to define the perceptual modifications in the watermark embedding process. Lin et al. [16] suggested a watermarking method depending on the rendering conditions of the 3D images. Another blind diverse watermarking method was suggested in [17] based on the DBIR method performed on the centre image and the depth image generated by the content provider. Kim et al. [18] also introduced a watermarking method for 3D DBIR images through the utilization of the quantization on Dual-Tree Complex Wavelet Transform (DT-CWT) coefficients. To improve the watermark robustness, two features of the DT-CWT are utilized, which are the approximate shift invariance and the directional selectivity. In [19], some efficient and robust hybrid watermarking schemes for different color image systems have been presented.

An efficient watermarking method for 3D images based on DBIR scheme was presented in [20] by utilizing the Scale-Invariant Feature Transform (SIFT) to choose some suitable regions for watermarking and the spread spectrum technique to insert the watermark data in the DCT coefficients of the selected regions. A 3DV blind watermarking scheme based on a virtual view invariant domain was introduced in [21]. The luminance average values of the 3DV frames are chosen for watermark embedding. In [22], another 3DV watermarking scheme that concentrates on perceptual quality embedding was introduced. It takes advantage of motion on the z-axis, visual features, and the rendered hidden pixels from the depth data.

Swati et al. [23] suggested a fragile watermarking method, where the watermark is inserted in the Least Significant Bit (LSB) of the non-zero quantized coefficients in the HEVC compressed video. Ogawa et al. [24] proposed an efficient watermarking scheme for HEVC bit streams that inserts the watermark information through the video compression phase. Also, there are several traditional works existing for the watermarking of the 2D H.264/AVC compressed bit streams. Zhang et al. [25] suggested a video watermarking scheme, where the security information is represented in a preprocessed binary data sequence and embedded into the middle frequency coefficients in the I frame. To enhance the watermark verification, the coefficients signs are altered depending on the watermark. The work introduced in [25] has been enhanced in [26] by concentrating on gray-scale characters and patterns. Qiu et al. [27] suggested a robust intra-frame watermark embedding scheme in quantized DCT coefficients and a fragile inter-frame watermark embedding method in motion vectors. Kuo and Lo [28] enhanced the video watermark embedding scheme that was suggested in [27] by selecting more appropriate regions for both robust and fragile watermark embedding within the H.264 compressed video through the video encoding process.

In [29], the process of watermark embedding is executed through directly changing some data bits within the bit stream, however the pre-embedding process has complex computations. In [30], the same authors of [29] suggested a non-blind and robust watermarking method by utilizing the Watson Visual Model (WVM) for watermark embedding in the I frame. Their proposed non-blind method [30] was 
extended for the P frame in [31], where the watermark bits are embedded in all non-zero coefficients of the $\mathrm{P}$ frame. An information hiding model was implemented in [32] to choose the watermark embedding area based on the forbidden-zone-data-hiding concepts. The sign parity of the coefficients and the values of the middle-frequency coefficients are altered for watermark embedding in the I frame [33]. In [34], the watermark has been embedded in the non-zero coefficients of the P-frame in the compressed domain to achieve better perceptual quality of the watermarked video frames and a minimal increase in video bit rate. In [35], a structure preserving non-blind H.264 watermarking scheme was suggested to insert watermarks through substituting secret bits in the motion vector differences of the non-reference images. Su et al. [36] suggested another non-blind watermark embedding algorithm for the I frames and P frames. The watermark embedding is implemented based on the spread spectrum technique and the WVM [31]. Generally, there are few contributions in the literature on 3D compressed video watermarking.

It is noticed that several authors introduced a lot of works on 3D image watermarking in the spatial domain. Most recent 3D image and video watermarking techniques have been implemented in transform domains. Generally, there are few contributions in the literature on 3D compressed video watermarking techniques. Some of these introduced techniques have critical problems with watermark verification and extraction. The traditional video watermarking techniques have not achieved adequate watermarked and extracted watermark subjective and objective qualities in the presence of multimedia attacks. Thus, they have low robustness and imperceptibility. Moreover, most of the traditional video watermarking techniques work on un-compressed images. So, they require more computations in the watermark insertion and extraction processes, where a complete encoding and decoding of the transmitted stream are needed for embedding and extraction of the watermark data. Thus, they increase the computational overhead. Furthermore, the traditional video watermarking techniques failed in selecting the most suitable regions inside the host frames for watermark embedding. Thus, they have an effect on the imperceptibility and quality of the watermarked frames, and they also increase the computations.

Taking into account the limitations of the state-of-the-art video watermarking techniques, the main contribution of this paper is to present efficient hybrid techniques for secure 3DV communication. These proposed hybrid techniques efficiently protect the copyright of the 3DV streams to preserve both robustness and imperceptibility. Therefore, they have good imperceptibility, high quality, high robustness, acceptable bit rates, low computational complexity, and adequate immunity to different types of multimedia attacks compared to the traditional techniques.

\section{3DV Prediction Structure (3DV-PS)}

The 2DV coding differs greatly from the 3DV coding, which benefits from the high inter-view matching amongst different views, and also from space-temporal correlations among frames within the self-same view video [37]. The predictive 3DV coding framework is presented in Fig.1 [38]. It comprises the inter- and intra-frames. The Disparity Compensation Prediction (DCP) and Motion Compensation Prediction (MCP) are used at the encoder to achieve high 3DV compression. In the 3DV-PS shown in Fig.1, the MCP is utilized to determine the MVs among different frames in the same 
video view and the DCP is used to estimate the DVs among various frames of contiguous views. So, each frame in the 3DV-PS can be estimated through temporally neighboring frames and/or through various view frames.

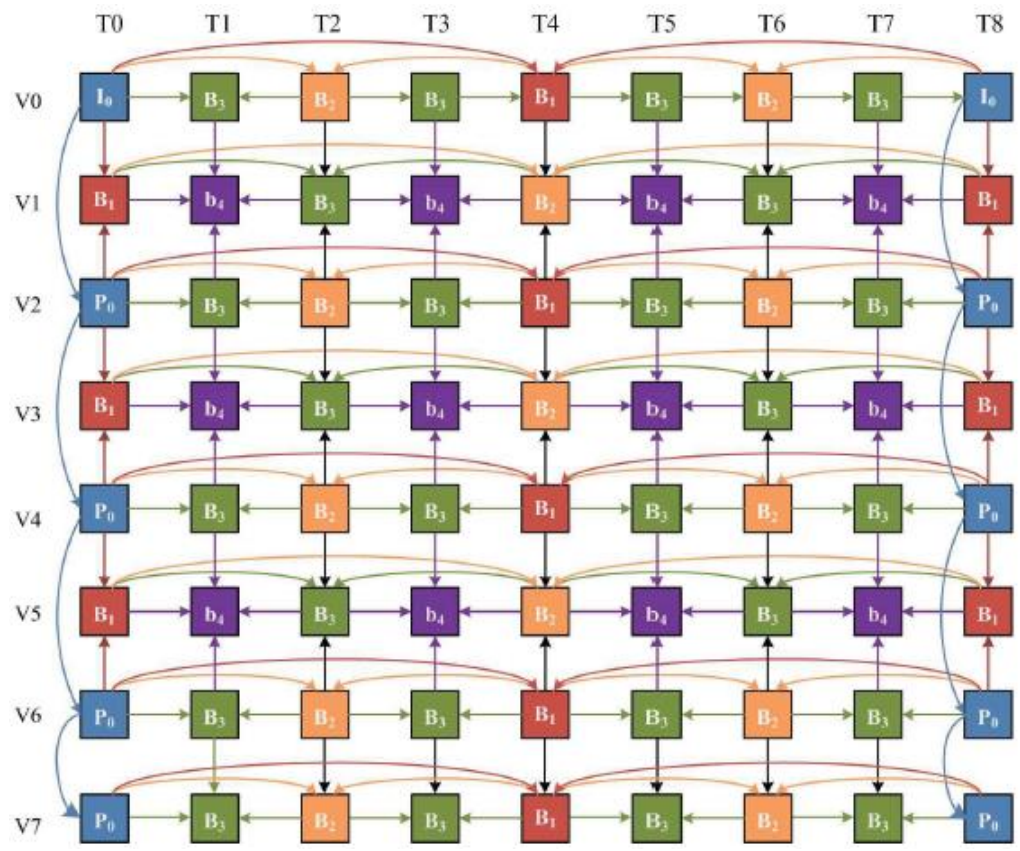

Fig. $13 \mathrm{D}$ video prediction coding model.

The 3DV-PS has an efficacious coding/decoding achievement. It introduces a Group Of Pictures (GOPs) that contains eight different time pictures. The vertical direction represents multiple camera views and the horizontal direction represents the temporal axis. For example, the V0 view is coded only via time correlation depending on the MCP. The other even V2, V4, and V6 views are likewise coded based on the MCP. On the other hand, the prime key frames are coded via the inter-view predictions depending on the DCP. In the V1, V3, and V5 odd views, the inter-view and temporal estimations (DCP+MCP) are simultaneously used for enhancing the coding performance. In this paper, the $3 \mathrm{DV}$ view is referred to considering its elementary locality 3DV frame. Therefore, as presented in Fig.1, the odd views are called B-views, the even views are referred to as P-views, and the V0 view is symbolized as I-view. The final view might be even or odd based on the suggested 3DV GOPs. In this paper, it is proposed to be a P-view. The 3DV-PS presents two encoded frames type: the P and B interframes and the I intra-frames. The inter frames in the B-views are estimated via the intra-frames inside the I-view, and also from the inter-frames within the P-views. Thence, if an error occurs in the I frames or in the $\mathrm{P}$ frames, it propagates to the relative inter-view frames and furthermore to the adjacent temporal frames in the same video view.

\section{MC-CDMA System Model}

Direct Sequence Code Division Multiple Access (DS-CDMA) is a scheme to share spectrum amongst multiple simultaneous users. Furthermore, it can employ frequency diversity, using RAKE receivers. But, in a dispersive multipath channel, DS-CDMA with a spread factor $N$ can accommodate $N$ simultaneous users only if highly complex interference cancellation techniques are used. Therefore, 
in DS-CDMA systems, the narrowband message signal is multiplied by a very large-bandwidth signal called the spreading signal. The chip rate of the code signal must be much higher than the bit rate of the information signal. All users in a DS-CDMA system use the same carrier frequency and may transmit, simultaneously. Each user has his own spreading signal, which is approximately orthogonal to the spreading signals of all other users. The receiver despreads the signal using the same code. It has to be able to synchronize the received signal with the locally generated code, otherwise, the original signal cannot be recovered. Unfortunately, it is difficult to implement it practically. So, MC-CDMA can handle $N$ simultaneous users with good BER, using standard receiver techniques. MC-CDMA is used to avoid excessive bit-errors on subcarriers that have a deep fade. Thus, the number of subcarriers required is larger than the number of symbols or bits transported simultaneously. MC-CDMA exchanges this encoder by an $N \times N$ matrix process. So, MC-CDMA is the transmission scheme of future mobiles because of its bandwidth efficiency and inherent diversity over a fading channel. However, the multi-carrier signals show highly varying envelope power waveforms, which hinder the popular employment of multi-carrier CDMA. But, MC-CDMA and OFDM are resilient to InterSymbol Interference (ISI) and spectrally efficient. Thus, MC-CDMA possesses both merits of DSCDMA and OFDM.

Generally, multi-carrier modulation is considered as a key ingredient in future, beyond $3 \mathrm{G}$ and $4 \mathrm{G}$ communication systems. In particular, combining the merits of Orthogonal Frequency Division Multiplexing (OFDM) and CDMA in MC-CDMA. However, it suffers from Multiple Access Interference (MAI) in a multiuser setting, which leads to a decrease in the overall Bit Error Rate (BER) performance abruptly with the increase in the number of users. Multi-User Detection (MUD) techniques have been introduced to mitigate MAI and improve the system performance [39]. The effects of fading channels on the transmitted data with MC-CDMA are much more severe than the effects of AWGN channels. So, there is a need for a powerful interleaving mechanism to combat these effects.

The basic MC-CDMA signal is generated by a serial concatenation of classical DS-CDMA and OFDM. Each chip of the direct sequence spread data symbol is mapped onto a different sub-carrier. Thus, with MC-CDMA, the chips of a spread data symbol are transported in parallel on various subcarriers, in contrast to a serial transmission with DS-CDMA. The number of simultaneously active users in an MC-CDMA mobile radio system is $K$ [39].

Figure 2 shows multi-carrier spectrum spreading of one complex-valued data symbol $d^{(k)}$ assigned to user $k$. The rate of the serial data symbols is $1 / T_{d}$. For brevity, but without loss of generality, the MC-CDMA signal generation is described for a single data symbol per user as far as possible, so that the data symbol index can be omitted. At the transmitter, the complex-valued data symbol $d^{(k)}$ is multiplied by the user specific spreading code [39]:

$$
\mathbf{c}^{(k)}=\left(\mathbf{c}_{0}^{(k)}, \mathbf{c}_{1}^{(k)}, \ldots, \mathbf{c}_{N-1}^{(k)}\right)^{T}
$$

of length $N$. The chip rate of the serial spreading code $\mathbf{c}^{(k)}$ before serial-to-parallel conversion is,

$$
\frac{1}{T_{c}}=\frac{N}{T_{d}}
$$


and it is $N$ times higher than the data symbol rate $\frac{1}{T_{d}}$. The complex-valued sequence obtained after spreading is given in vector notations by [39]:

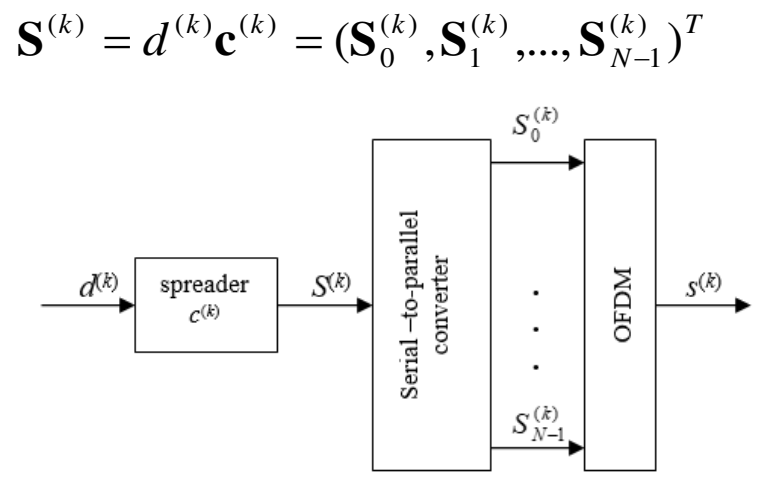

Fig. 2 Multi-carrier spread spectrum signal generation.

A multi-carrier spread spectrum signal is obtained after modulating the components $\mathbf{S}_{n}^{(k)}, n=0, \ldots$, $N-1$, in parallel onto $N$ sub-carriers. With multi-carrier spread spectrum, each data symbol is spread over $N-1$ sub-carriers. In cases where the number of sub-carriers $N_{c}$ of one OFDM symbol is equal to the spreading code length $N$, the OFDM symbol duration with multi-carrier spread spectrum including a guard interval results in $T_{s}^{\prime}=T_{g}+N T_{c}$. In this case, one data symbol per user is transmitted in one OFDM symbol. Thus, the use of spreading codes in MC-CDMA adds an anti-jamming capability to CDMA. MC-CDMA has a good resistance to ISI like OFDM.

In the synchronous downlink, it is computationally efficient to add the spread signals of the $K$ users before the OFDM operation as depicted in Fig. 3. The superposition of the $K$ sequences $\mathbf{S}^{(k)}$ results in the sequence [39]:

$$
\mathbf{S}=\sum_{k=0}^{K-1} \mathbf{S}^{(k)}=\left(\mathbf{S}_{0}, \mathbf{S}_{1}, \ldots, \mathbf{S}_{N-1}\right)^{T}
$$

In matrix form, $S$ can be rewritten as follows:

$$
\mathbf{S}=\mathbf{C d}
$$

where

$$
\mathbf{d}=\left(d^{(0)}, d^{(1)}, \ldots, d^{(K-1)}\right)^{T}
$$

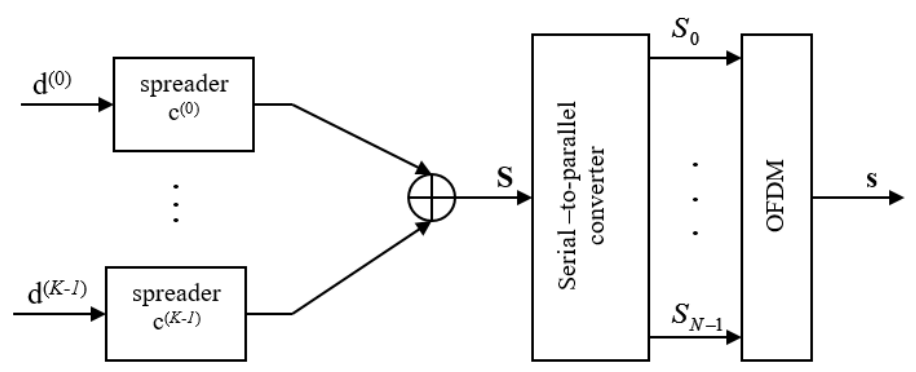

Fig. 3 MC-CDMA transmitter.

The received vector of the transmitted sequence $\mathbf{s}$ after OFDM demodulation and frequency deinterleaving is given by: 


$$
R^{\prime}=\mathbf{H s}+\mathbf{n}=\left(R_{0}, R_{1}, \ldots, R_{N-1}\right)^{T}
$$

where $\mathbf{H}$ is the $N \times N$ channel matrix and $\boldsymbol{n}$ is the noise vector of length $N$. The vector $R^{\prime}$ is fed to the data detector in order to get a hard or soft estimate of the transmitted data. The data detection needs to begin with an equalizer.

\section{Proposed Joint 3DV Transmission Framework}

In this section, the proposed 3DV transmission system is introduced. The general framework of the proposed 3DV communication process is shown in Fig. 4. The proposed joint 3DV transmission system model to transmit the (DWT+SVD) watermarked 3DV frames over MC-CDMA wireless channel consists of four main stages as indicated in Fig. 4. The first stage is (DWT+SVD)-based 3DV watermarking. The second stage is chaotic map interleaving and convolution coding applied on the binary watermarked 3DV data. The third stage is the spreading, scrambling, and OFDM modulation. The final stage is the LMMSE equalization process.

Firstly, the huge amount of the 3DV data is encoded using the 3D H.264/MVC or H.265/HEVC encoder to reduce the data size to be compatible with the transmission requirements over limitedresources wireless channels. Then, the encoded 3DV frames are watermarked with the proposed DWT+SVD watermarking process. After that, the resultant compressed watermarked 3DV frames are converted to a binary data format. Then, chaotic interleaving is applied to the binary information prior to the modulation process. The chaotic interleaving is used to mitigate the wireless OFDM and MCCDMA induced problems and to reduce the wireless channel effects on the transmitted bit streams, and also to add a degree of encryption to the transmitted 3DV compressed watermarked frames. In this paper, the compressed 3D data is transmitted over a wireless MC-CDMA system. Therefore, due to the high compression of the transmitted 3DV sequence, it becomes more sensitive to wireless channel errors. So, we propose the application of a chaotic Baker map interleaving technique at the transmitter and the application of an efficient equalization technique at the receiver for efficient transmission of compressed watermarked 3DV frames over the MC-CDMA wireless channel.

Therefore, in the proposed 3DV transmission framework presented in Fig. 4, the 3DV sequences are firstly compressed using the compression stage based on employing the MVC/HEVC codecs which exploit the intra- and inter-view correlations between the 3DV frames. Then, the resulting compressed 3DV frames are watermarked using the watermarking stage based on the suggested DWT and SVD techniques. Moreover, the resultant compressed 3D video watermarked frames are transformed to binary format in an $M \times M$ square binary matrix form. After that, chaotic Baker map interleaving is applied on the resultant binary format of the 3DV data. After this interleaving process, the square binary matrix is reshaped again to its original dimensions. Then, the OFDM modulation is employed on the binary data.

At the receiver side, after performing the OFDM demodulation step, the channel estimation is performed assuming that the receiver has a complete knowledge of the secret key of the chaotic map. After that, the received signal is equalized to suppress the ISI resulting from the communication channel. So, the equalization stage is implemented at the receiver side to mitigate the wireless channel effects on the transmitted 3DV data. Furthermore, after the equalization step, the chaotic de- 
randomization process is applied after reshaping the binary data into a square binary matrix. Then, the square binary matrix is reshaped again into its original dimensions. The compressed 3D video watermarked frames are retrieved from the binary data. Then, the compressed 3D video frames are recovered from the watermarked 3D video compressed frames after applying the proposed extraction process. Finally, estimates of the original 3DV frames are retrieved and recovered by employing the MVC/HEVC decoding process.

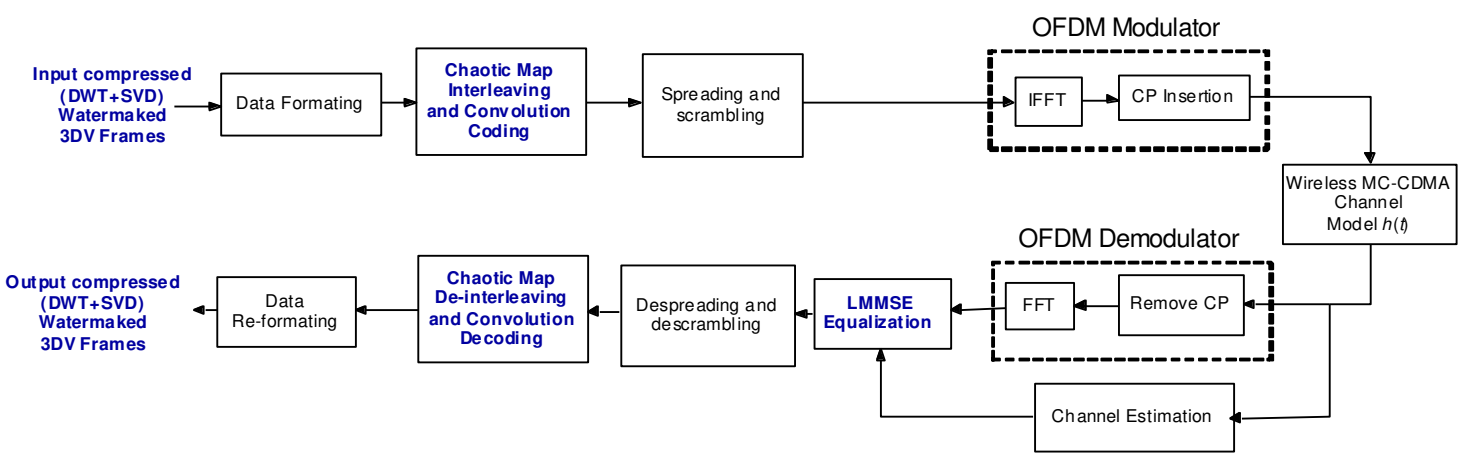

Fig. 4 Block diagram of the proposed joint framework for compressed (DWT+SVD) watermarked 3DV transmission over wireless MC-CDMA system.

The steps of the suggested joint 3DV transmission framework are explained as follows:

1. Perform the compression MVC/HEVC process on the transported 3DV frames.

2. Perform the proposed (DWT+SVD) embedding process on the transmitted compressed 3DV frames.

3. Convert each compressed (DWT+SVD) watermarked 3DV frame to the binary matrix form.

4. Reshape the binary matrix, which is non-square, to an $M \times M$ square binary matrix format.

5. Apply the proposed chaotic Baker map interleaving on the reshaped square binary matrix.

6. Reshape the square binary matrix to its original dimensions.

7. Perform the convolution coding, spreading, scrambling, and OFDM modulation on the binary data.

8. Transmit the binary modulated data over the wireless MC-CDMA channel.

9. Apply the OFDM demodulation at the receiver to estimate the channel, assuming that the receiver has used the same secret key of the chaotic map, which is used at the transmitter.

10. Perform the equalization, dispreading, descrambling, and convolution decoding process on the received signal to suppress the ISI. The proposed equalizer is the LMMSE equalizer that can be described as follows [12]:

$$
\hat{\mathbf{d}}=\left(\mathbf{H}_{d}^{H} \mathbf{H}_{d}+\alpha \mathbf{I}\right)^{-1} \mathbf{H}_{d}^{H} \mathbf{r}
$$

11. Reshape the binary data to a square matrix and apply the chaotic de-interleaving on binary matrix.

12. Reshape the square binary matrix to its original dimensions.

13. Retrieve the compressed (DWT+SVD) watermarked video frames.

14. Perform an (DWT+SVD) extraction process to restore the compressed 3DV frames and watermarks.

15. Perform a decoding MVC/HEVC process to restore the original 3D video frames.

In the following subsections, more details about the proposed DWT+SVD watermarking, chaotic baker map interleaving, and LMMSE equalization techniques are presented below. 


\subsection{Proposed (DWT+SVD)-Based 3DV Watermarking}

In the proposed (DWT+SVD)-based watermarking of 3D video frames, the DWT is used on the cover and watermark frames to produce four non-overlapping multi-resolution coefficient groups $\mathrm{HH}$, LH, HL, LL for the cover and watermark frames using 2-D filters in each dimension. The filters divide the input cover or watermark frame into four non-overlapping multi-resolution sub bands, a lower resolution approximation image (LL1), horizontal (HL1), vertical (LH1), and diagonal (HH1) detail components. The process is repeated to obtain multiple scale wavelet decomposition of the cover and watermark frames. Then, the SVD is utilized on the LL component to give two orthogonal matrices $\mathbf{U}$, $\mathbf{V}$ and one diagonal matrix $\mathbf{S}$. The watermark $\mathbf{W}$ is added into the matrix $\mathbf{S}$ of the host $\mathrm{I}$-frames or $\mathbf{P}$ and B-frames, and then a new SVD process is performed on the new matrix $\mathbf{S}+k \mathbf{W}$ to get $\mathbf{U}_{\mathbf{w}}, \mathbf{S}_{\mathbf{w}}$ and $\mathbf{V}_{\mathbf{w}}$, where $k$ is a scale factor that controls the strength of the watermark embedding to the original 3DV frame. The watermarked $3 \mathrm{DV}$ frame $\mathbf{F}_{\mathbf{w}}$ is obtained by multiplying the matrices $\mathbf{U}, \mathbf{S}_{\mathbf{w}}$, and $\mathbf{V}^{\mathrm{T}}$. The stages of the proposed (DWT+SVD)-based 3DV watermarking algorithm are described below.

\subsubsection{Proposed DWT+SVD Watermark Embedding Process}

The steps of DWT+SVD watermark embedding are introduced as follows:

1. The original compressed 3DV is partitioned into groups of $M$ frames.

2. Every frame into the group is converted from the RGB into the $Y_{b} C_{r}$ color space and just the luminance values $\mathrm{Y}$ of the frames is further processed.

3. Every luminance frame is transformed into the DWT domain using Wavelet decomposition with $L=2$ resolution levels.

4. The SVD is performed on the 2D matrix of selected wavelet detail coefficients of the luminance $\mathrm{Y}$ of each frame of the group $j$ of frames $\left(\mathbf{B}_{i}(j)\right.$ matrix $)$ of the original compressed 3DV to obtain the $\operatorname{SVs}\left(\mathbf{S}_{i}(j)\right.$ matrix), where $i=1,2,3, \ldots, M$, and $M$ is the number of frames.

$$
\mathbf{B}_{i}(j)=\mathbf{U}_{i}(j) \cdot \mathbf{S}_{i}(j) \cdot \mathbf{V}_{i}^{T}(j)
$$

5. Add the watermark ( $\mathbf{W}$ matrix) to the $\mathbf{S}_{i}(j)$ matrix of each video frame of the group $j$ of frames.

$$
\mathbf{D}_{i}(j)=\mathbf{S}_{i}(j)+k \mathbf{W}(j)
$$

6. Employ SVD on each $\mathbf{D}_{i}(j)$ matrix of each video frame of the group $j$ of frames to get the SVs of each one of the group $j$ of frames $\left(\mathbf{S}_{w i}(j)\right.$ matrix $)$.

$$
\mathbf{D}_{i}(j)=\mathbf{U}_{w i}(j) \cdot \mathbf{S}_{w i}(j) \cdot \mathbf{V}_{w i}^{T}(j)
$$

7. Use the $\left(\mathbf{S}_{w i}(j)\right.$ matrix $)$ of each one of the group $j$ of frames to build the watermarked frames.

$$
\mathbf{B}_{w i}(j)=\mathbf{U}_{i}(j) \cdot \mathbf{S}_{w i}(j) \cdot \mathbf{V}_{i}^{T}(j)
$$

8. After the entire watermark has been embedded, the Inverse Discrete Wavelet Transform (IDWT) is computed to obtain the watermarked video frames. 


\subsubsection{Proposed DWT+SVD Watermark Extraction Process}

Suppose that we have received a distorted 3DV and $\mathbf{U}_{w i}, \mathbf{V}_{w i}, \mathbf{S}_{i}$, matrices. The steps to extract the corrupted watermark are summarized as follows:

1. The obtained compressed watermarked $3 \mathrm{DV}$ is divided into groups of $M$ frames.

2. Every frame of the group is converted from the RGB into the $\mathrm{YC}_{\mathrm{b}} \mathrm{C}_{\mathrm{r}}$ color space.

3. Every luminance frame is transformed into the DWT domain using Wavelet decomposition with $L=2$ resolution levels.

4. Apply SVD on each possibly distorted watermarked frame of the group $j$ of frames $\left(\mathbf{B}_{w i}^{*}(j)\right.$ matrix) to get the SVs of each one $\mathbf{S}_{w i}^{*}(j)$ matrix.

$$
\mathbf{B}_{w i}^{*}(j)=\mathbf{U}_{i}(j) \cdot \mathbf{S}_{w i}^{*}(j) \cdot \mathbf{V}_{i}^{T}(j)
$$

5. Extract the matrices that contain the watermark of each frame of the group $j$ of frames using the $\mathbf{U}_{w i}(j), \mathbf{V}_{w i}(j), \mathbf{S}_{w i}^{*}(j)$ matrices.

$$
\mathbf{D}_{i}(j)=\mathbf{U}_{w i}(j) \cdot \mathbf{S}_{w i}^{*}(j) \cdot \mathbf{V}_{w i}(j)
$$

6. From every frame of a group of $M$ frames, recover the corrupted watermarks ( $\mathbf{W}_{i}^{*}$ matrix) from the $\mathbf{D}_{i}$ matrices.

$$
\frac{\mathbf{D}_{i}(j)-\mathbf{S}_{i}(j)}{k}=\mathbf{W}_{i}^{*}
$$

\subsection{Proposed Chaotic Baker Map Interleaving Process}

The chaotic map is suggested as an interleaving technique. The Baker map is one of the most common and efficient types of the chaotic interleaving maps, which generates a square matrix in a permuted version. The Baker map is used to randomize the square matrix of data in its discretized form which represents an $M \times M$ matrix as follows [11]:

$$
B(r, s)=\left[\frac{M}{n_{i}}\left(r-M_{i}\right)+s \bmod \left(\frac{M}{n_{i}}\right), \frac{n_{i}}{M}\left(s-s \bmod \left(\frac{M}{n_{i}}\right)\right)+M_{i}\right]
$$

where the new indices of the data item are $B(r, s)$ at $(r, s), M_{i} \leq r<M_{i}+n_{i}, 0<s<M$ and $M_{i}=n_{1}+$ $n_{2}+\ldots+n_{i}$. Figure 5 shows an example of the proposed chaotic interleaving of an $(8 \times 8)$ square matrix, which uses the secret key, $\mathrm{S}_{k e y}=\left(n_{1}, n_{2}, n_{3}\right)=(2,4,2)$.

The proposed chaotic baker map interleaving steps are explained as follows:

1. Divide the square matrix $M \times M$ into $k$ vertical rectangles of width $n_{i}$ and height $M$.

2. Stretch vertical rectangles in the horizontal direction and after that contract them vertically to obtain an $n_{i} \times M$ horizontal rectangle.

3. Perform a stack on horizontal rectangles as shown in Fig. 5-a, where the right one is put at the top and the left one is put at the bottom.

4. Divide each output $n_{i} \times M$ vertical rectangle into $n_{i}$ boxes, whose dimensions are $M=n_{i} \times n_{i}$ and exactly contain $M$ points.

5. Map each box column by column into a row as shown in Fig. 5-b. 


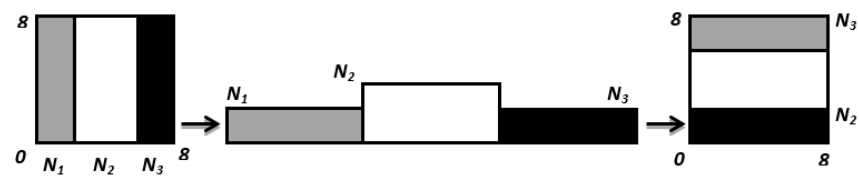

(a)

$N_{1}=2 \quad N_{2}=4 \quad N_{3}=2$

\begin{tabular}{|cc|cccc|cc}
\hline$Y_{1}$ & $Y_{2}$ & $Y_{3}$ & $Y_{4}$ & $Y_{5}$ & $Y_{6}$ & $Y_{7}$ & $Y_{8}$ \\
$Y_{9}$ & $Y_{10}$ & $Y_{11}$ & $Y_{12}$ & $Y_{13}$ & $Y_{14}$ & $Y_{15}$ & $Y_{16}$ \\
$Y_{17}$ & $Y_{18}$ & $Y_{19}$ & $Y_{20}$ & $Y_{21}$ & $Y_{22}$ & $Y_{23}$ & $Y_{24}$ \\
$Y_{25}$ & $Y_{26}$ & $Y_{27}$ & $Y_{28}$ & $Y_{29}$ & $Y_{30}$ & $Y_{31}$ & $Y_{32}$ \\
\cline { 1 - 4 }$Y_{33}$ & $Y_{34}$ & $Y_{35}$ & $Y_{36}$ & $Y_{37}$ & $Y_{38}$ & $Y_{39}$ & $Y_{40}$ \\
$Y_{41}$ & $Y_{42}$ & $Y_{43}$ & $Y_{44}$ & $Y_{45}$ & $Y_{46}$ & $Y_{47}$ & $Y_{48}$ \\
$Y_{49}$ & $Y_{50}$ & $Y_{51}$ & $Y_{52}$ & $Y_{53}$ & $Y_{54}$ & $Y_{55}$ & $Y_{56}$ \\
\cline { 4 - 5 }$Y_{57}$ & $Y_{58}$ & $Y_{59}$ & $Y_{60}$ & $Y_{61}$ & $Y_{62}$ & $Y_{63}$ & $Y_{64}$ \\
& & & & & & &
\end{tabular}

PM Output

(b)

\begin{tabular}{|llllllll|}
\hline$Y_{31}$ & $Y_{23}$ & $Y_{15}$ & $Y_{7}$ & $Y_{32}$ & $Y_{24}$ & $Y_{16}$ & $Y_{8}$ \\
\hline$Y_{63}$ & $Y_{55}$ & $Y_{47}$ & $Y_{39}$ & $Y_{64}$ & $Y_{56}$ & $Y_{48}$ & $Y_{40}$ \\
\hline$Y_{11}$ & $Y_{3}$ & $Y_{12}$ & $Y_{4}$ & $Y_{13}$ & $Y_{5}$ & $Y_{14}$ & $Y_{6}$ \\
\hline$Y_{27}$ & $Y_{19}$ & $Y_{28}$ & $Y_{20}$ & $Y_{29}$ & $Y_{21}$ & $Y_{30}$ & $Y_{22}$ \\
\hline$Y_{43}$ & $Y_{35}$ & $Y_{44}$ & $Y_{36}$ & $Y_{45}$ & $Y_{37}$ & $Y_{46}$ & $Y_{38}$ \\
\hline$Y_{59}$ & $Y_{51}$ & $Y_{60}$ & $Y_{52}$ & $Y_{61}$ & $Y_{53}$ & $Y_{32}$ & $Y_{54}$ \\
\hline$Y_{25}$ & $Y_{17}$ & $Y_{9}$ & $Y_{1}$ & $Y_{26}$ & $Y_{18}$ & $Y_{10}$ & $Y_{2}$ \\
\hline$Y_{57}$ & $Y_{49}$ & $Y_{41}$ & $Y_{33}$ & $Y_{58}$ & $Y_{50}$ & $Y_{42}$ & $Y_{34}$ \\
\hline
\end{tabular}

CMR Output

Fig. 5 Chaotic interleaving example using a secret $k e y=(2,4,2)$ : (a) Discretize Baker map, (b) An $8 \times 8$ randomizations output.

\subsection{Proposed Convolution Coder}

The proposed convolution code is based on the Viterbi algorithm [40]. The proposed convolution coder presents redundant bits into the stream of the transmitted data through the use of linear shift registers as shown in Fig. 6. The introduced redundant bits are used to detect and correct the accumulated errors in the received data bit streams at the receiver.

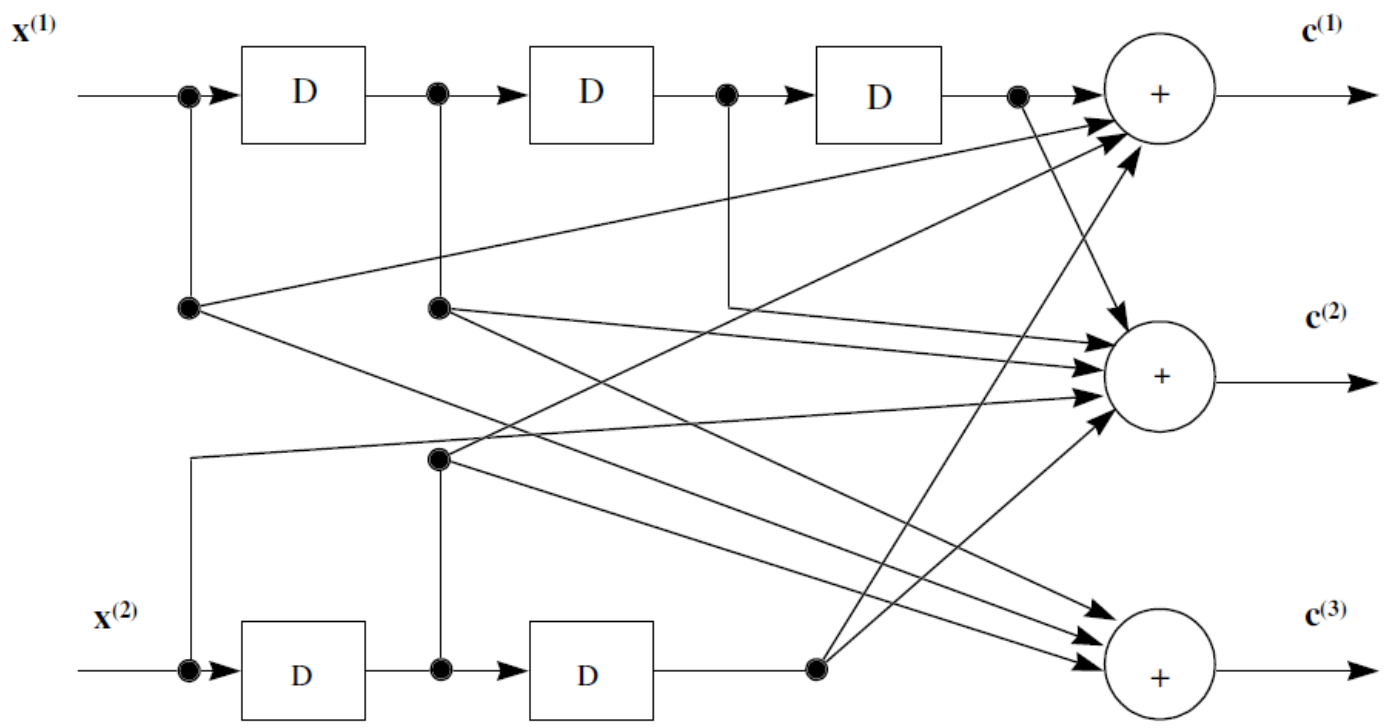

Fig. 6 A proposed convolution encoder, where $x^{(i)}$ is an input information bit stream and $c^{(i)}$ is an output encoded bit stream. 


\subsection{Proposed Channel LMMSE Equalizer}

To eliminate the ISI caused by the multipath fading channel and to improve the overall performance of the wireless communication system, an efficient linear equalization technique must be used. Linear equalization has different types in frequency domain such as Zero Forcing (ZF), Regularized Zero Forcing (RZF), and Linear Minimum Mean Square Error (LMMSE) equalization. The ZF solution is defined as [41]:

$$
\mathbf{W}_{Z F}=\left(\mathbf{H}^{H} \mathbf{H}\right)^{-1} \mathbf{H}^{H}
$$

where $\mathbf{H}$ is the channel matrix. The advantages of the frequency domain $\mathrm{ZF}$ equalizer is that the source data of the equalizer and the statistics of the additive noise are not required. The disadvantages of the frequency domain $\mathrm{ZF}$ equalizer are that it causes noise enhancement and its computations for matrix inversion are time consuming. A new regularization part is added into Eq. (17) to avoid noise enhancement in the ZF equalizer output as follows [41]:

$$
\mathbf{W}_{R Z F}=\left(\mathbf{H}^{H} \mathbf{H}+\alpha \mathbf{I}\right)^{-1} \mathbf{H}^{H}
$$

where $\alpha$ is a regularization parameter. The resulting equalizer is called RZF equalizer. So, in the RZF equalizer, the additive noise and the statistics of the transmitted data are not required. This leads to a better equalizer, which eliminates the Mean Square Error (MSE) and removes the ISI. When $\alpha=1 / \mathrm{SNR}$, the equalizer is equivalent to the LMMSE equalizer. The LMMSE is defined as:

$$
\mathbf{W}_{\text {LMMSE }}=\left(\mathbf{H}^{H} \mathbf{H}+\frac{1}{S N R} \mathbf{I}\right)^{-1} \mathbf{H}^{H}
$$

\section{Simulation Results and Comparative Analysis}

The performance of the proposed (DWT+SVD) watermarking technique is determined using the evaluation metrics of the correlation coefficient $\left(c_{r}\right)$ between the original watermark and the extracted watermark and the PSNR to measure the quality of the reconstructed (DWT+SVD) watermarked 3DV frames at the receiver. The PSNR is the ratio between the maximum power of a signal and the power of corrupting noise. It is usually expressed in decibel scale. The Mean Square Error (MSE) is the squared error between the recovered and the original (DWT+SVD) watermarked 3DV frame. The MSE, PSNR, and $c_{r}$ are given as follows:

$$
\begin{gathered}
M S E=\frac{1}{M N} \sum_{y=1}^{M} \sum_{x=1}^{N}\left[I(x, y)-I^{\prime}(x, y)\right]^{2} \\
P S N R=20 * \log \left(\frac{255}{\sqrt{M S E}}\right) \\
C_{r}=\frac{\mathbf{W}^{*} \cdot \mathbf{W}}{\left\|\mathbf{W}^{*}\right\| \cdot\|\mathbf{W}\|}
\end{gathered}
$$

where the original (DWT+SVD) watermarked $3 \mathrm{DV}$ frame is $I(x, y)$, the reconstructed (DWT+SVD) watermarked $3 \mathrm{DV}$ frame is $I^{\prime}(x, y)$, and the dimensions of the (DWT+SVD) watermarked video frame 
are $M \times N$. The $\mathbf{W}$ is the original watermark and $\mathbf{W}^{*}$ is the extracted corrupted watermark. To evaluate the performance and efficiency of the proposed hybrid techniques, different standardized 3D video test sequences with different motion activities and spatial resolutions of $1024 \times 768$ (Newspaper) and 1920×1088 (Shark and Poznan_Hall2) are used [42] as inputs to the simulation framework. We carried out several simulation experiments at different Signal-to-Noise Ratio (SNRs) of 10dB, 15dB, and 20dB to test the performance of the proposed hybrid schemes. The model of SUI-3 wireless channel is used in our simulation. It is one model of six channel models adopted by the IEEE 802.16a standard, which are used for evaluating the performance of broadband wireless systems in the 2-11 GHz band [43]. The SUI-3 channel model has three Rayleigh fading taps at delays of 0, 0.5, and $1 \mathrm{~ms}$ and relative powers of $0 \mathrm{~dB},-5 \mathrm{~dB}$, and $-10 \mathrm{~dB}$, respectively. In our experiments, the encoded $3 \mathrm{D}$ tested watermarked MVC/HEVC frames are transmitted over the MC-CDMA system model with only convolution coding and equalization $[11,40,41]$ to compare with the proposed hybrid framework of chaotic interleaving, convolution coding, and equalization techniques. Also, we compared the proposed hybrid techniques with the case of no employing chaotic interleaving, convolution coding, and equalization techniques. The employed simulation parameters are summarized in Table (1).

Table 1 Simulation parameters.

\begin{tabular}{|c|c|c|}
\hline \multirow{4}{*}{ Transmitter } & Modulation & BPSK \\
\cline { 2 - 3 } & Video size & $1024 \times 768$ and $1920 \times 1088$ \\
\cline { 2 - 3 } & Cyclic prefix & 20 samples \\
\cline { 2 - 3 } & Transmitter IFFT size & M=256 symbols \\
\cline { 2 - 3 } Channel & Chaotic map size & $16 \times 16$ \\
\cline { 2 - 3 } & Environment of noise & SUI-3 channel \\
\hline \multirow{2}{*}{ Receiver } & Equalization & AWGN \\
\cline { 2 - 3 } & Estimation of channel & PMMSE \\
\hline
\end{tabular}

In our experiments, the compressed (DWT+SVD) watermarked 3D tested video frames are transmitted over the MC-CDMA system with employing the joint framework of (chaotic map interleaving + convolution coding + LMMSE equalization). The original selected host frames, the original watermark frames, and the (DWT+SVD) watermarked frames in the case of error free channel of the tested 3DV sequences are shown in Fig. 7. Figures 8 to 10 show a comparison between the case of utilizing the LMMSE equalization and convolution coding with and without chaotic interleaving and the case of not using any of them to transmit the compressed (DWT+SVD) watermarked 3DV Newspaper frames over a wireless MC-CDMA channel at different SNRs of 10dB, 15dB, and $20 \mathrm{~dB}$. Figures 8.a and 8.d show the received watermarked frame with PSNR=12.5138dB, and its extracted watermark frame with the correlation coefficient between extracted watermark and the original watermark $\left(c_{r}\right)$ equal to 0.7115 in the case of not using the interleaving, convolution coding, and LMMSE equalization at $\mathrm{SNR}=10 \mathrm{~dB}$. On the other hand, Figures 8.b and 8.e introduce the received watermarked frame with PSNR $=34.5289 \mathrm{~dB}$ and its extracted watermark frame with $c_{r}=0.9923$ in the state of not using the interleaving and with employing the convolution coding and LMMSE equalization only at $\mathrm{SNR}=10 \mathrm{~dB}$. Figures 8.c and 8.f present the received watermarked frame with $\mathrm{PSNR}=35.7235 \mathrm{~dB}$ and its extracted watermark frame with $c_{r}=0.9947$ in the state of exploiting the chaotic map interleaving jointly with the convolution coding and LMMSE equalization at SNR=10dB. 
Figures 9.a and 9.d introduce the received watermarked frame with PSNR $=12.7757 \mathrm{~dB}$ and its extracted watermark frame with $c_{r}=0.7953$, in the case of not using the interleaving, convolution coding, and LMMSE equalization at $\mathrm{SNR}=15 \mathrm{~dB}$. Figures 9.b and 9.e show the received watermarked frame with PSNR=47.3455dB and its extracted watermark frame with $c_{r}=0.9999$ in the case of not using the interleaving and with employing the convolution coding and LMMSE equalization only at $\mathrm{SNR}=15 \mathrm{~dB}$. Figures 9.c and 9.f present the received watermarked frame with $\mathrm{PSNR}=50.3125 \mathrm{~dB}$ and its extracted watermark frame with $c_{r}=0.9999$ in the state of exploiting the chaotic map interleaving in addition to using convolution coding and LMMSE equalization at $\mathrm{SNR}=15 \mathrm{~dB}$.

Figures 10.a and 10.d show the received watermarked frame with PSNR $=12.8567 \mathrm{~dB}$ and its extracted watermark frame with $c_{r}=0.8469$ in the case of not exploiting the interleaving, convolution coding, and LMMSE equalization at SNR=20dB. On the other hand, Figures 10.b and 10.e introduce the received watermarked frame with $\mathrm{PSNR}=54.1503 \mathrm{~dB}$ and its extracted watermark frame with $c_{r}=0.9999$ in the case of not using the interleaving and with employing the convolution coding and LMMSE equalization only at SNR=20dB. Figures 10.c and 10.f present the received watermarked frame with PSNR $=60.1720 \mathrm{~dB}$ and its extracted watermark frame with $c_{r}=0.9999$ in the state of employing the chaotic map interleaving jointly with the convolution coding and LMMSE equalization at $\mathrm{SNR}=20 \mathrm{~dB}$.

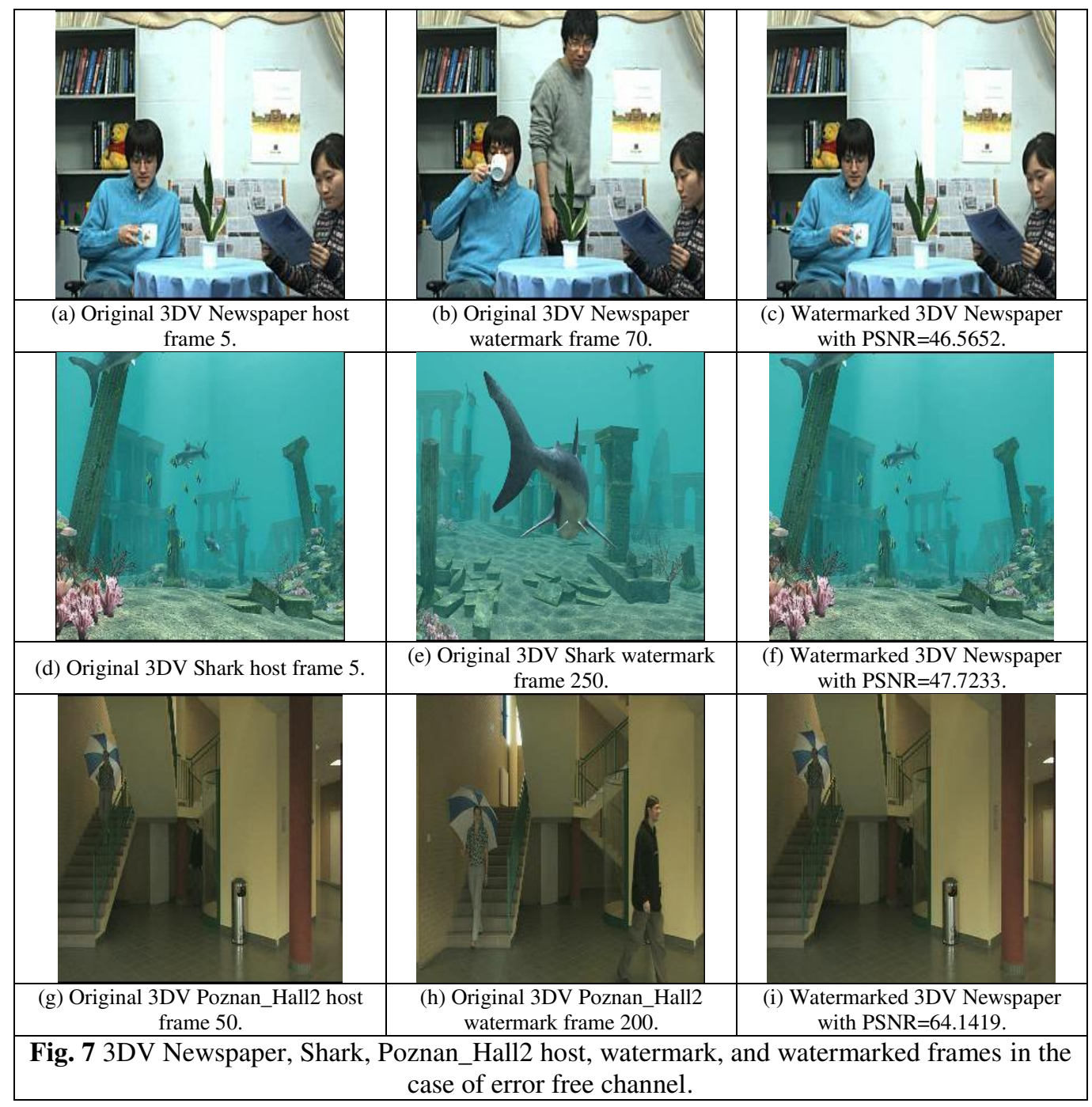



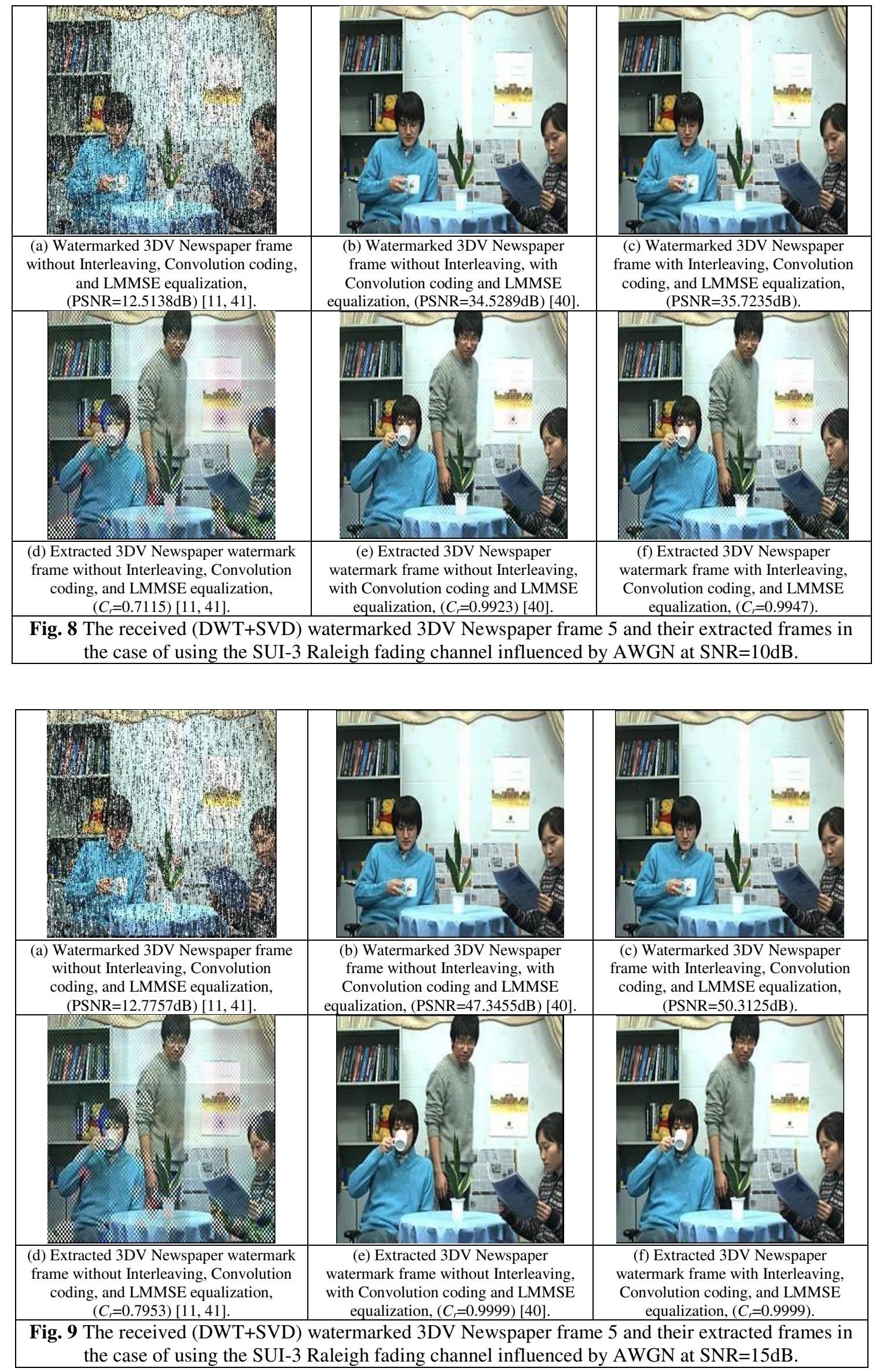

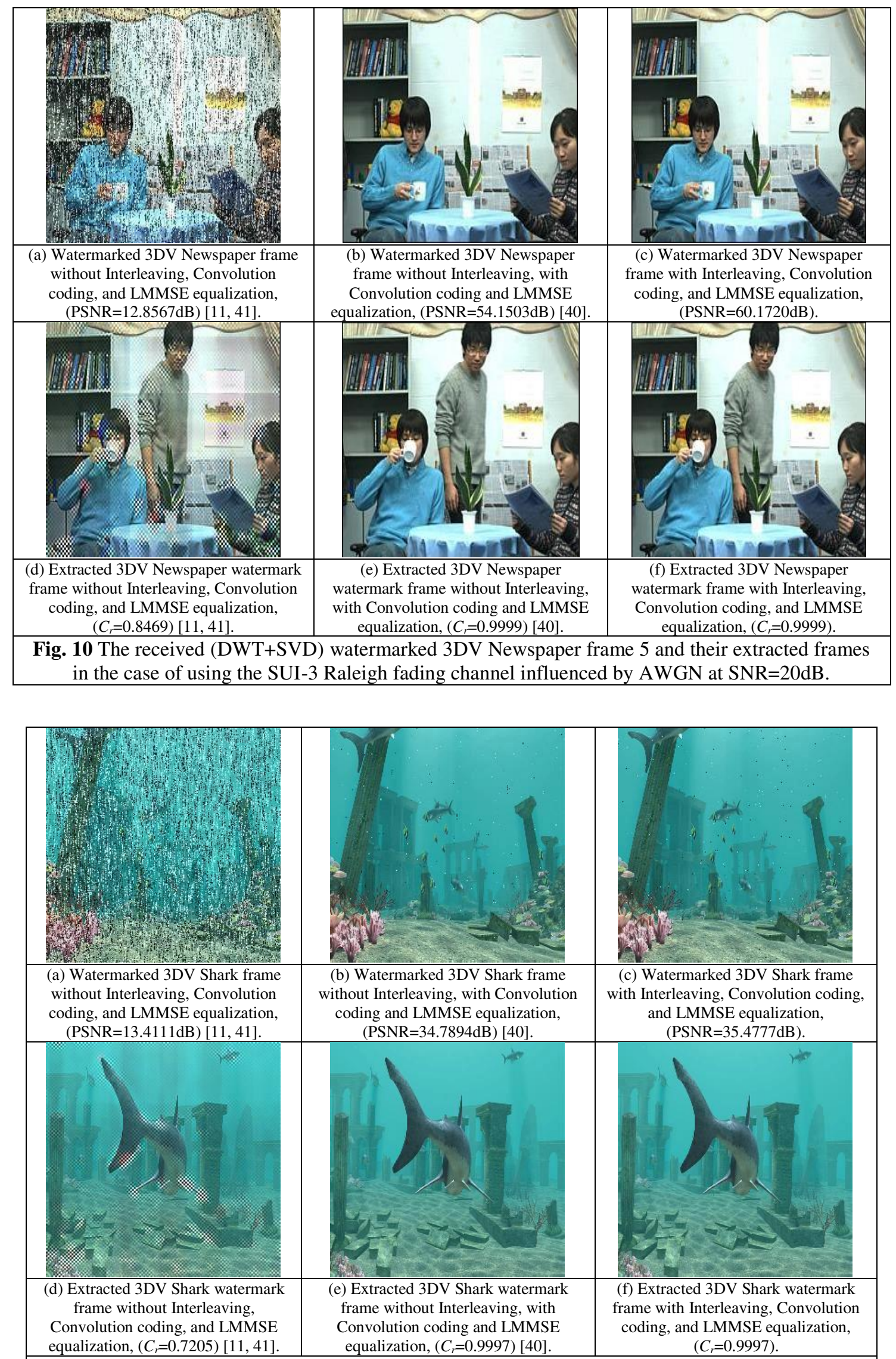

Fig. 11 The received (DWT+SVD) watermarked 3DV Shark frame 5 and their extracted frames in the case of using the SUI-3 Raleigh fading channel influenced by AWGN at SNR=10dB. 


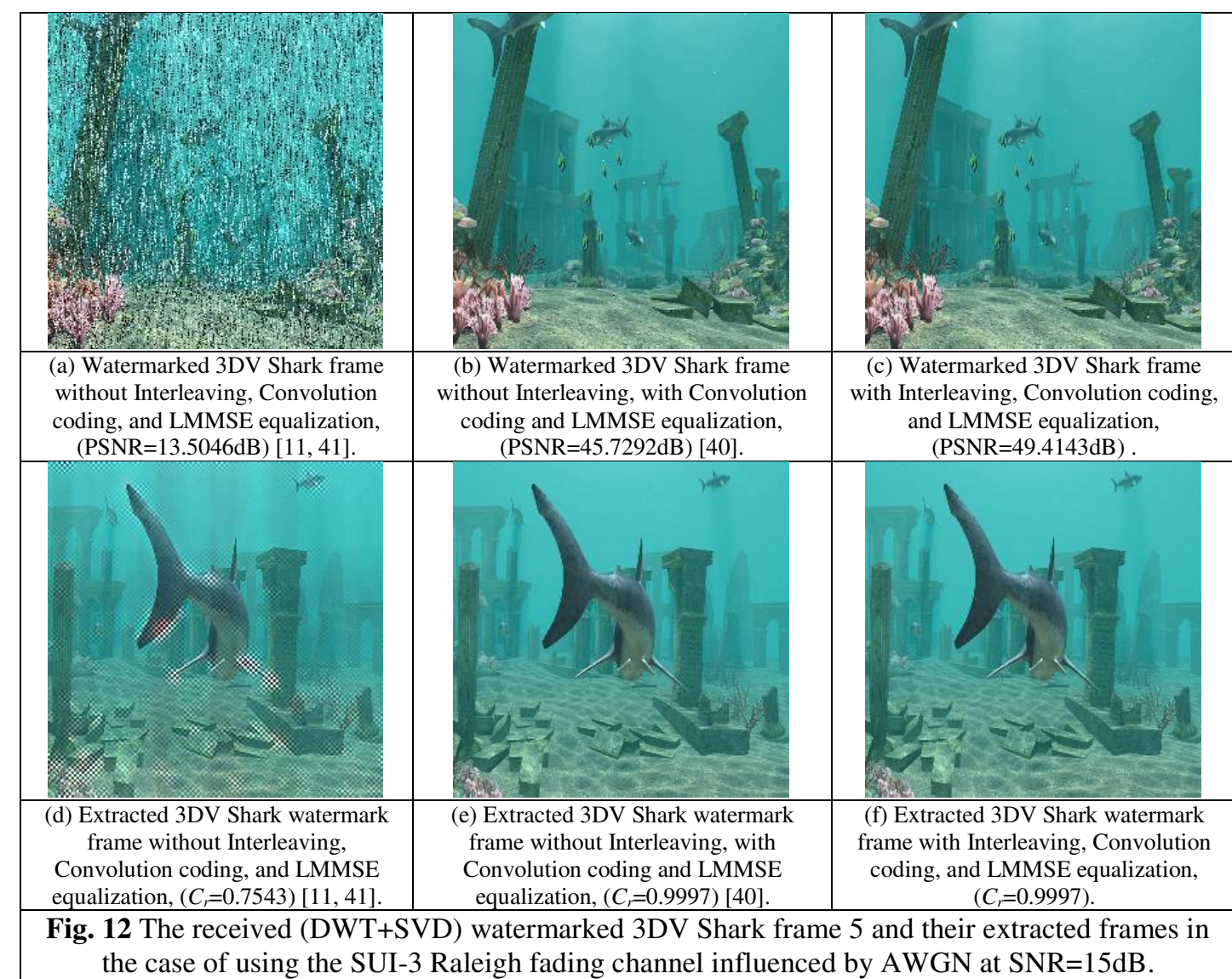

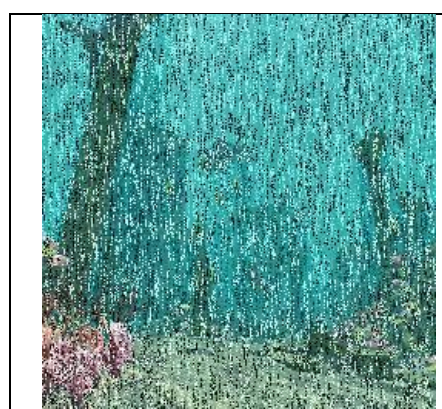

(a) Watermarked 3DV Shark frame without Interleaving, Convolution coding, and LMMSE equalization, $(\mathrm{PSNR}=13.5311 \mathrm{~dB})[11,41]$

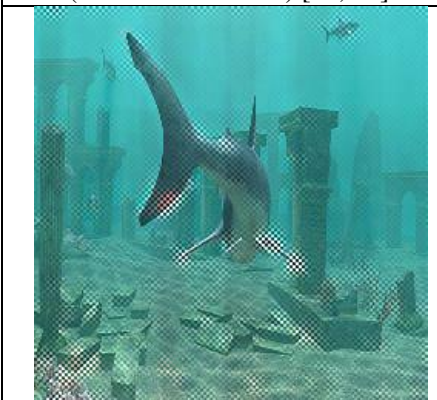

(d) Extracted 3DV Shark watermark frame without Interleaving,

Convolution coding, and LMMSE equalization, $\left(C_{r}=0.7878\right)[11,41]$.

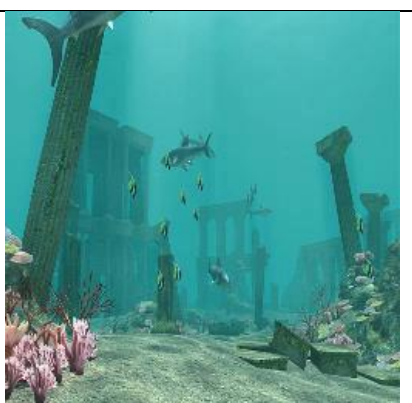

(b) Watermarked 3DV Shark frame without Interleaving, with Convolution coding and LMMSE equalization, $(\mathrm{PSNR}=52.4342 \mathrm{~dB})[40]$.

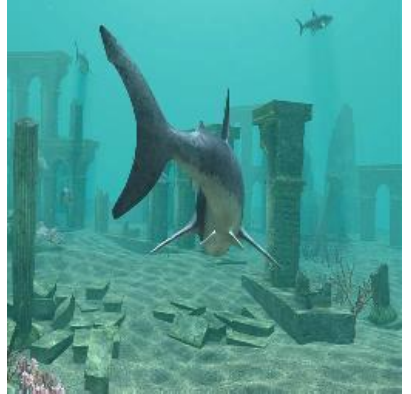

(e) Extracted 3DV Shark watermark frame without Interleaving, with Convolution coding and LMMSE equalization, $\left(C_{r}=0.9998\right)$ [40].

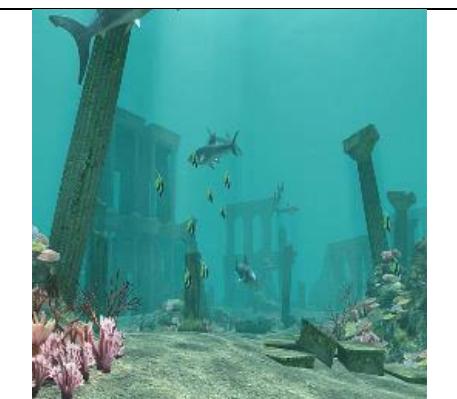

(c) Watermarked 3DV Shark frame with Interleaving, Convolution coding, and LMMSE equalization, $(\mathrm{PSNR}=58.2338 \mathrm{~dB})$

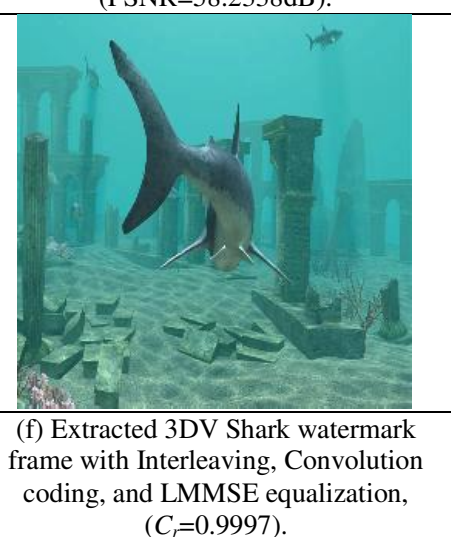

Fig. 13 The received (DWT+SVD) watermarked 3DV Shark frame 5 and their extracted frames in the case of using the SUI-3 Raleigh fading channel influenced by AWGN at SNR=20dB. 


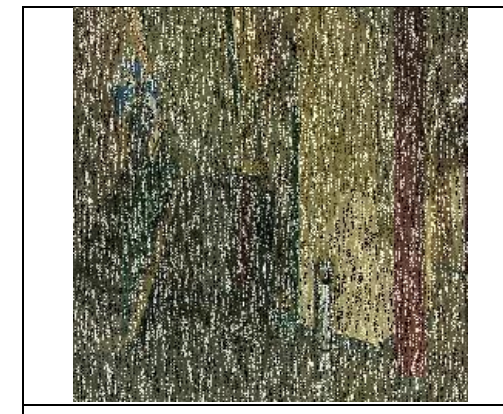

(a) Watermarked 3DV Poznan_Hall2 frame without Interleaving, Convolution coding, and LMMSE equalization, $(\mathrm{PSNR}=13.0004 \mathrm{~dB})[11,41]$.

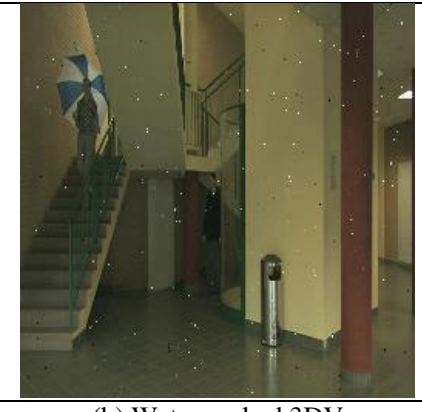

(b) Watermarked 3DV Poznan_Hall2 frame without Interleaving, with Convolution coding and LMMSE equalization, $(\mathrm{PSNR}=34.0880 \mathrm{~dB})$ [40].

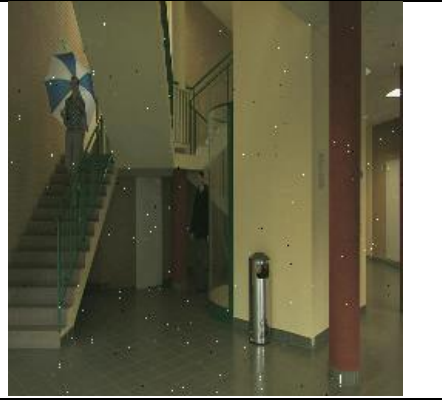

(c) Watermarked 3DV Poznan_Hall2 frame with Interleaving, Convolution coding, and LMMSE equalization, $(\mathrm{PSNR}=34.4085 \mathrm{~dB})$.

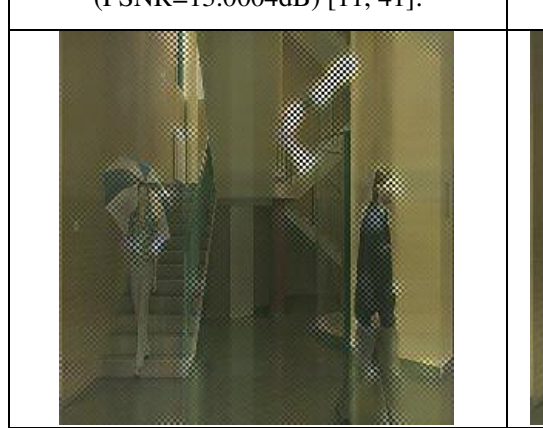

(d) Extracted 3DV Poznan_Hall2 watermark frame without Interleaving, Convolution coding, and LMMSE equalization, $\left(C_{r}=0.4883\right)[11,41]$.

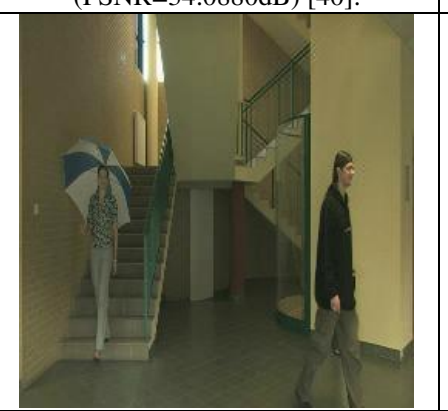

(e) Extracted 3DV Poznan_Hall2 watermark frame without Interleaving, with Convolution coding and LMMSE equalization, $\left(C_{r}=0.9987\right)$ [40].

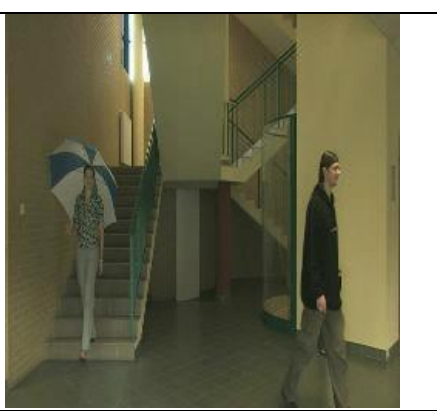

(f) Extracted 3DV Poznan_Hall2 watermark frame with Interleaving, Convolution coding, and LMMSE equalization, $\left(C_{r}=0.9987\right)$.

Fig. 14 The received (DWT+SVD) watermarked 3DV Poznan_Hall2 frame 50 and their extracted frames in the case of using the SUI-3 Raleigh fading channel influenced by AWGN at SNR=10dB.

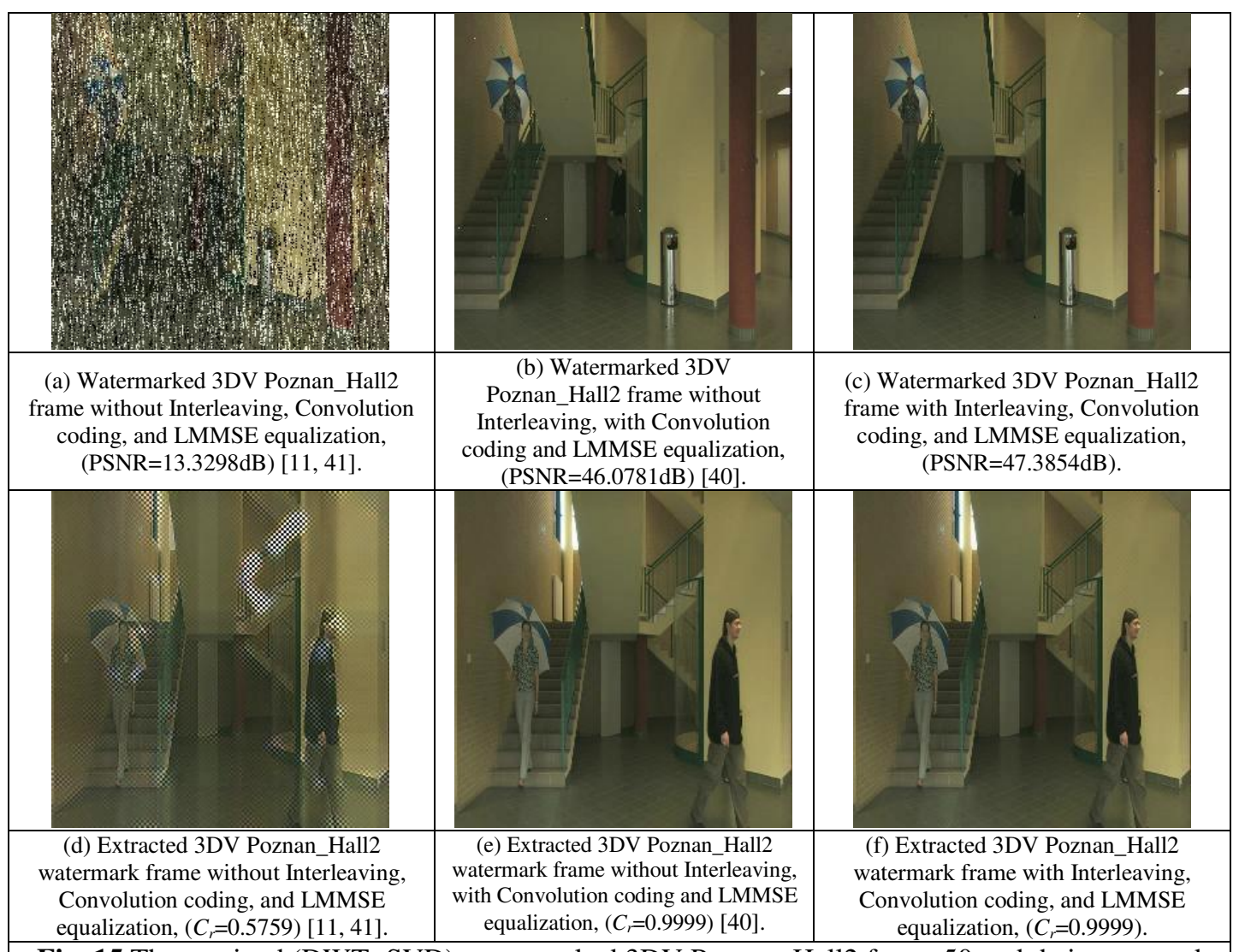

Fig. 15 The received (DWT+SVD) watermarked 3DV Poznan_Hall2 frame 50 and their extracted frames in the case of using the SUI-3 Raleigh fading channel influenced by AWGN at SNR=15dB. 


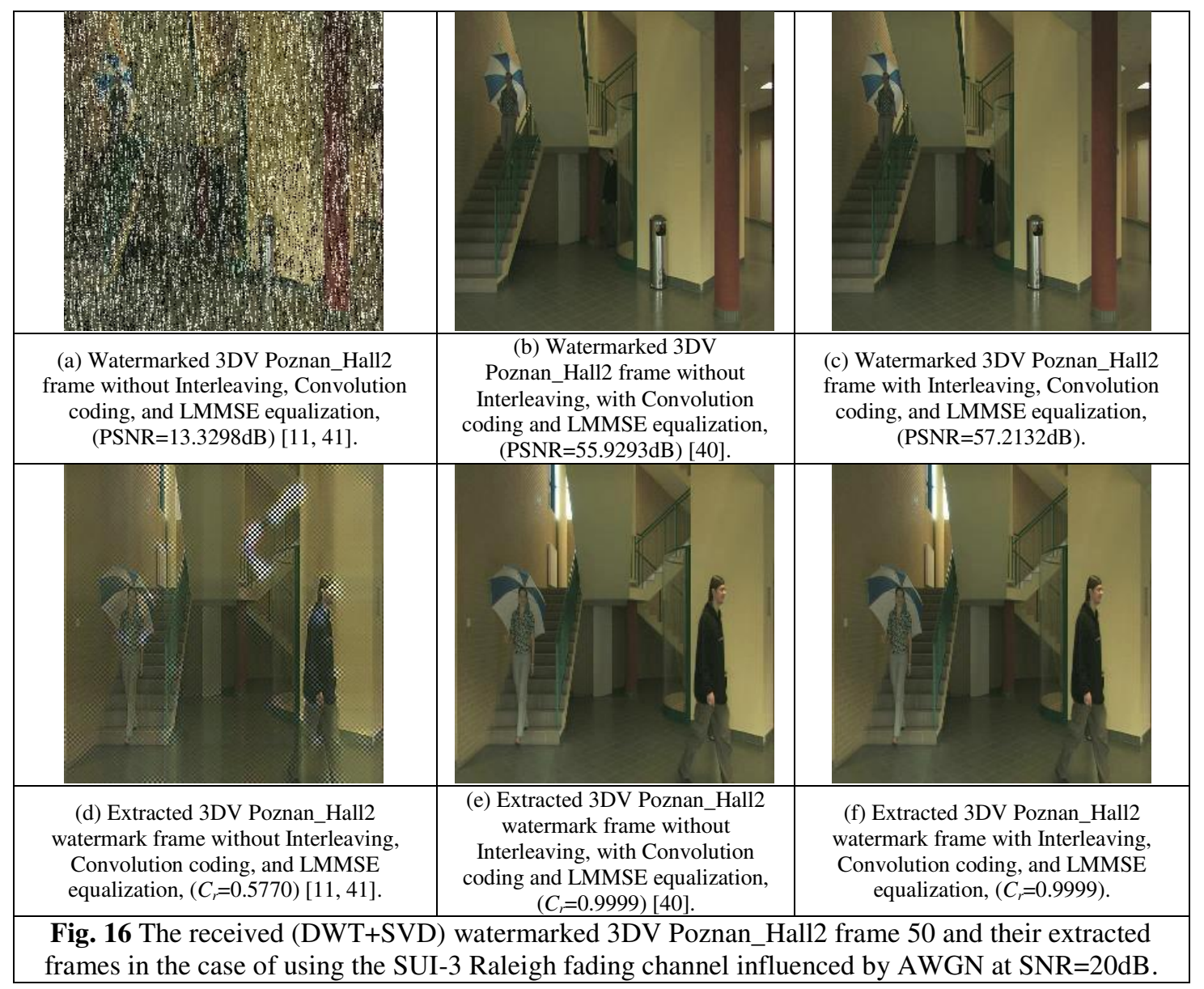

In order to confirm the performance enhancement and efficiency of the proposed joint techniques, we have run more simulation tests on the Shark and Poznan_Hall2 3DV test sequences, which have various spatial resolutions and temporal characteristics. Figures 11 to 16 give comparison results of the Shark and Poznan_Hall2 sequences in the case of employing (the convolution coding and LMMSE equalization with and without chaotic map interleaving) compared with the case of not exploiting any of them to transmit the compressed (DWT+SVD) watermarked 3DV frames efficiently over wireless MC-CDMA system at different SNRs of $10 \mathrm{~dB}, 15 \mathrm{~dB}$, and $20 \mathrm{~dB}$. From all the introduced simulation results, it is confirmed that employing the chaotic map interleaving jointly with convolution coding and linear equalization enhances the wireless transmission of compressed 3DV watermarked frames. Also, they reduce the effect of channel noise and thus increase the maximum correlation coefficient between extracted watermarks and original watermarks.

To further clarify the efficiency of the proposed hybrid watermarking scheme in protecting and securing the transmitted compressed 3DV bit streams over MC-CDMA wireless channel, we have compared its performance, robustness, and imperceptibility with those of the state-of-the-art watermarking schemes such as DCT, SVD, DWT, DCT+SVD, and DCT+DWT [19, 44, 45]. Table 2 presents the objective average PSNR results of the watermarked frames and the average $C_{r}$ results of the extracted watermark frames for the Newspaper, Shark, and Poznan_Hall2 3DV streams with the proposed embedding scheme compared to the state-of-the-art embedding schemes in the absence of attacks. It is clear that the proposed watermarking scheme achieves high average PSNR and $C_{r}$ values for all tested 3DV frames compared to those of the related works. 
Table 2 Objective average PSNR values of the watermarked frames and average $C_{r}$ values of the extracted watermark frames for the Newspaper, Shark, and Poznan_Hall2 3DV streams in the absence of attack.

\begin{tabular}{|c|c|c|c|}
\hline \multirow{2}{*}{$\begin{array}{c}\text { Watermarking } \\
\text { Technique }\end{array}$} & \multicolumn{3}{|c|}{ PSNR $(\mathbf{d B}) / \boldsymbol{C}_{\boldsymbol{r}}$} \\
\cline { 2 - 4 } & Newspaper & Shark & Poznan_Hall2 \\
\hline DWT+SVD (Proposed) & $\mathbf{4 9 . 8 1 1 1 / 0 . 9 9 8 9}$ & $\mathbf{4 8 . 2 7 0 3 / 0 . 9 9 9 9}$ & $\mathbf{4 7 . 3 5 6 2 / 0 . 9 9 8 9}$ \\
\hline DWT [19] & $26.9775 / 0.7865$ & $27.7775 / 0.7549$ & $26.6582 / 0.7954$ \\
\hline SVD [44] & $25.0839 / 0.6598$ & $25.7451 / 0.7632$ & $25.7024 / 0.6984$ \\
\hline DCT [45] & $24.0852 / 0.6985$ & $25.7170 / 0.6874$ & $23.9895 / 0.6745$ \\
\hline DCT+DWT [45] & $27.0208 / 0.7021$ & $29.9522 / 0.7798$ & $28.4567 / 0.8021$ \\
\hline DCT+SVD [19] & $31.4677 / 0.8125$ & $32.7205 / 0.8254$ & $30.0358 / 0.8924$ \\
\hline
\end{tabular}

Also, to further verify the performance efficiency of the proposed hybrid watermarking scheme in protecting and securing the transmitted compressed 3DV bit streams over MC-CDMA wireless channel against different multimedia attacks, we have compared the performance, robustness, and imperceptibility of the proposed hybrid watermarking scheme with those of the state-of-the-art watermarking schemes such as DCT, SVD, DWT, DCT+SVD, and DCT+DWT [19, 44, 45] in the presence of HEVC compression, rotation, Gaussian noise, blurring, JPEG compression, resizing, and crop attacks. Table 3 to 9 present the objective average PSNR results of the watermarked frames and the average $C_{r}$ results of the extracted watermark frames for the Newspaper, Shark, and Poznan_Hall2 3DV streams with the proposed embedding scheme compared to the state-of-the-art embedding schemes in the presence of HEVC compression, rotation, Gaussian noise, blurring, JPEG compression, resizing, and crop attacks. From the simulation results Table 3 to 9 in the case of different multimedia attacks, it is noticed that the proposed hybrid watermarking technique gives not only the highest PSNR values between the original and watermarked frames, but also the best correlation values between the original and extracted watermarks. So, it is clear that the proposed DWT + SVD technique achieves the best results in the case of different multimedia attacks.

Table 3 Objective average PSNR values of the watermarked frames and average $C_{r}$ values of the extracted watermark frames for the Newspaper, Shark, and Poznan_Hall2 3DV streams in the presence of HEVC compression attack.

\begin{tabular}{|c|c|c|c|}
\hline \multirow{2}{*}{$\begin{array}{c}\text { Watermarking } \\
\text { Technique }\end{array}$} & \multicolumn{3}{|c|}{ PSNR $(\mathbf{d B}) / \boldsymbol{C}_{\boldsymbol{r}}$} \\
\cline { 2 - 4 } & HEVC compression attack \\
\cline { 2 - 4 } & Newspaper & Shark & Poznan_Hall2 \\
\hline DWT+SVD (Proposed) & $\mathbf{4 2 . 7 5 6 1 / 0 . 9 8 9 7}$ & $\mathbf{4 1 . 3 2 6 4 / 0 . 9 8 8 9}$ & $\mathbf{4 1 . 0 3 2 4 / 0 . 9 8 7 9}$ \\
\hline DWT [19] & $22.5689 / 0.6879$ & $22.6895 / 0.6547$ & $21.7365 / 0.6759$ \\
\hline SVD [44] & $21.1235 / 0.5698$ & $21.6985 / 0.6997$ & $20.8024 / 0.5998$ \\
\hline DCT [45] & $19.2015 / 0.5967$ & $20.8243 / 0.5968$ & $21.8957 / 0.5978$ \\
\hline DCT+DWT [45] & $23.0324 / 0.6897$ & $24.8657 / 0.6984$ & $23.5634 / 0.6986$ \\
\hline DCT+SVD [19] & $25.5167 / 0.7230$ & $26.8395 / 0.7586$ & $25.3624 / 0.7023$ \\
\hline
\end{tabular}

Table 4 Objective average PSNR values of the watermarked frames and average $C_{r}$ values of the extracted watermark frames for the Newspaper, Shark, and Poznan_Hall2 3DV streams in the presence of rotation attack.

\begin{tabular}{|c|c|c|c|}
\hline \multirow{2}{*}{$\begin{array}{c}\text { Watermarking } \\
\text { Technique }\end{array}$} & \multicolumn{3}{|c|}{ PSNR (dB) / $\boldsymbol{C}_{\boldsymbol{r}}$} \\
\cline { 2 - 4 } & \multicolumn{3}{|c|}{ Rotation attack } \\
\cline { 2 - 4 } & Newspaper & Shark & Poznan_Hall2 \\
\hline DWT+SVD (Proposed) & $\mathbf{2 5 . 6 2 4 3 / 0 . 9 7 6 5}$ & $\mathbf{2 6 . 8 6 7 4 / 0 . 9 7 2 4}$ & $\mathbf{2 5 . 4 3 7 2 / 0 . 9 7 3 9}$ \\
\hline DWT [19] & $21.6257 / 0.6964$ & $21.7264 / 0.6653$ & $20.6254 / 0.7021$ \\
\hline SVD [44] & $20.3581 / 0.6023$ & $20.5864 / 0.7105$ & $21.3762 / 0.6218$ \\
\hline DCT [45] & $20.4438 / 0.6847$ & $21.3547 / 0.6342$ & $22.9014 / 0.6294$ \\
\hline DCT+DWT [45] & $22.7308 / 0.7005$ & $23.8134 / 0.7153$ & $22.6308 / 0.6942$ \\
\hline DCT+SVD [19] & $24.2014 / 0.7138$ & $25.7985 / 0.7624$ & $24.4628 / 0.7438$ \\
\hline
\end{tabular}


Table 5 Objective average PSNR values of the watermarked frames and average $C_{r}$ values of the extracted watermark frames for the Newspaper, Shark, and Poznan_Hall2 3DV streams in the presence of Gaussian noise attack.

\begin{tabular}{|c|c|c|c|}
\hline \multirow{2}{*}{$\begin{array}{c}\text { Watermarking } \\
\text { Technique }\end{array}$} & \multicolumn{3}{|c|}{ PSNR (dB) / $\boldsymbol{C}_{\boldsymbol{r}}$} \\
\cline { 2 - 4 } & \multicolumn{3}{|c|}{ Gaussian noise attack } \\
\cline { 2 - 4 } & Newspaper & Shark & Poznan_Hall2 \\
\hline DWT+SVD (Proposed) & $\mathbf{3 7 . 8 5 4 6 / 0 . 9 7 7 8}$ & $\mathbf{3 6 . 4 5 3 7 / 0 . 9 8 9 4}$ & $\mathbf{3 6 . 3 8 6 4 / 0 . 9 7 8 6}$ \\
\hline DWT [19] & $20.6538 / 0.6834$ & $20.7126 / 0.6725$ & $20.8539 / 0.6820$ \\
\hline SVD [44] & $20.8697 / 0.6234$ & $20.9657 / 0.7108$ & $20.9768 / 0.6739$ \\
\hline DCT [45] & $20.5374 / 0.6723$ & $21.7694 / 0.6789$ & $22.7869 / 0.6759$ \\
\hline DCT+DWT [45] & $22.4356 / 0.7023$ & $22.7895 / 0.7194$ & $21.4452 / 0.6875$ \\
\hline DCT+SVD [19] & $24.8436 / 0.7129$ & $25.9124 / 0.7495$ & $24.7523 / 0.7268$ \\
\hline
\end{tabular}

Table 6 Objective average PSNR values of the watermarked frames and average $C_{r}$ values of the extracted watermark frames for the Newspaper, Shark, and Poznan_Hall2 3DV streams in the presence of blurring attack.

\begin{tabular}{|c|c|c|c|}
\hline \multirow{2}{*}{$\begin{array}{c}\text { Watermarking } \\
\text { Technique }\end{array}$} & \multicolumn{3}{|c|}{ PSNR (dB) / $\boldsymbol{C}_{\boldsymbol{r}}$} \\
\cline { 2 - 4 } & Blurring attack \\
\cline { 2 - 4 } & Newspaper & Shark & Poznan_Hall2 \\
\hline DWT+SVD (Proposed) & $\mathbf{3 9 . 8 6 4 7 / 0 . 9 9 2 0}$ & $\mathbf{3 8 . 5 3 4 6 / 0 . 9 9 1 4}$ & $\mathbf{4 0 . 2 4 7 5 / 0 . 9 8 9 9}$ \\
\hline DWT [19] & $21.6673 / 0.7023$ & $21.7542 / 0.6891$ & $21.4523 / 0.6937$ \\
\hline SVD [44] & $20.8695 / 0.6658$ & $20.7684 / 0.7086$ & $21.0124 / 0.6973$ \\
\hline DCT [45] & $20.4587 / 0.6985$ & $19.9475 / 0.7035$ & $20.3352 / 0.6638$ \\
\hline DCT+DWT [45] & $23.4215 / 0.7012$ & $23.7694 / 0.7248$ & $23.8953 / 0.7301$ \\
\hline DCT+SVD [19] & $24.9423 / 0.7689$ & $25.8874 / 0.7438$ & $24.6854 / 0.7642$ \\
\hline
\end{tabular}

Table 7 Objective average PSNR values of the watermarked frames and average $C_{r}$ values of the extracted watermark frames for the Newspaper, Shark, and Poznan_Hall2 3DV streams in the presence of JPEG compression attack.

\begin{tabular}{|c|c|c|c|}
\hline \multirow{2}{*}{$\begin{array}{c}\text { Watermarking } \\
\text { Technique }\end{array}$} & \multicolumn{3}{|c|}{ PSNR (dB) / $\boldsymbol{C}_{\boldsymbol{r}}$} \\
\cline { 2 - 4 } & \multicolumn{3}{|c|}{ JPEG compression attack } \\
\cline { 2 - 4 } & Newspaper & Shark & Poznan_Hall2 \\
\hline DWT+SVD (Proposed) & $\mathbf{4 1 . 8 7 1 6 / 0 . 9 8 8 6}$ & $\mathbf{4 0 . 5 4 2 1 / 0 . 9 9 0 4}$ & $\mathbf{4 0 . 9 6 4 3 / 0 . 9 8 6 9}$ \\
\hline DWT [19] & $22.6859 / 0.6975$ & $21.5894 / 0.6784$ & $21.9745 / 0.6920$ \\
\hline SVD [44] & $21.3624 / 0.6012$ & $21.7694 / 0.7062$ & $20.9207 / 0.6670$ \\
\hline DCT [45] & $19.3516 / 0.6348$ & $20.9407 / 0.6284$ & $21.9332 / 0.6098$ \\
\hline DCT+DWT [45] & $23.2436 / 0.7110$ & $23.9427 / 0.7438$ & $23.7624 / 0.7354$ \\
\hline DCT+SVD [19] & $24.6772 / 0.7456$ & $25.7986 / 0.7499$ & $24.4681 / 0.7256$ \\
\hline
\end{tabular}

Table 8 Objective average PSNR values of the watermarked frames and average $C_{r}$ values of the extracted watermark frames for the Newspaper, Shark, and Poznan_Hall2 3DV streams in the presence of resizing attack.

\begin{tabular}{|c|c|c|c|}
\hline \multirow{2}{*}{$\begin{array}{c}\text { Watermarking } \\
\text { Technique }\end{array}$} & \multicolumn{3}{|c|}{ PSNR (dB) / $\boldsymbol{C}_{\boldsymbol{r}}$} \\
\cline { 2 - 4 } & \multicolumn{3}{|c|}{ Resizing attack } \\
\cline { 2 - 4 } & Newspaper & Shark & Poznan_Hall2 \\
\hline DWT+SVD (Proposed) & $\mathbf{4 2 . 6 3 4 8 / 0 . 9 9 6 4}$ & $\mathbf{4 0 . 8 6 9 4 / 0 . 9 8 9 7}$ & $\mathbf{4 0 . 8 9 4 7 / 0 . 9 9 0 8}$ \\
\hline DWT [19] & $23.9675 / 0.7032$ & $22.7594 / 0.6859$ & $22.6956 / 0.6943$ \\
\hline SVD [44] & $21.6348 / 0.6635$ & $21.8764 / 0.7025$ & $21.1205 / 0.6512$ \\
\hline DCT [45] & $20.4536 / 0.6345$ & $21.2486 / 0.6570$ & $22.2947 / 0.6428$ \\
\hline DCT+DWT [45] & $22.6472 / 0.6924$ & $24.7468 / 0.7034$ & $24.1204 / 0.7068$ \\
\hline DCT+SVD [19] & $24.5568 / 0.7543$ & $25.7584 / 0.7684$ & $25.3094 / 0.7234$ \\
\hline
\end{tabular}

Table 9 Objective average PSNR values of the watermarked frames and average $C_{r}$ values of the extracted watermark frames for the Newspaper, Shark, and Poznan_Hall2 3DV streams in the presence of Cropping attack.

\begin{tabular}{|c|c|c|c|}
\hline \multirow{2}{*}{$\begin{array}{c}\text { Watermarking } \\
\text { Technique }\end{array}$} & \multicolumn{3}{|c|}{ PSNR (dB) / $\boldsymbol{C}_{\boldsymbol{r}}$} \\
\cline { 2 - 4 } & \multicolumn{3}{|c|}{ Cropping attack } \\
\cline { 2 - 4 } & Newspaper & Shark & Poznan_Hall2 \\
\hline DWT+SVD (Proposed) & $\mathbf{3 8 . 6 8 9 5 / 0 . 9 6 9 8}$ & $\mathbf{3 7 . 4 3 5 8 / 0 . 9 7 8 5}$ & $\mathbf{3 8 . 8 6 7 5 / 0 . 9 7 2 7}$ \\
\hline DWT [19] & $22.0347 / 0.6362$ & $21.5234 / 0.6739$ & $21.6345 / 0.6775$ \\
\hline SVD [44] & $20.8697 / 0.6635$ & $20.9986 / 0.7134$ & $20.5364 / 0.6430$ \\
\hline DCT [45] & $20.3657 / 0.6420$ & $21.7694 / 0.6532$ & $20.8694 / 0.6238$ \\
\hline DCT+DWT [45] & $22.8364 / 0.7032$ & $23.7249 / 0.7069$ & $23.1384 / 0.7155$ \\
\hline DCT+SVD [19] & $24.8669 / 0.7120$ & $25.5974 / 0.7362$ & $24.8964 / 0.6998$ \\
\hline
\end{tabular}




\section{Conclusions}

This paper presented an efficient compressed (DWT+SVD) watermarked three-dimensional video transmission system through wireless MC-CDMA channel using chaotic map interleaving with convolution coding and linear equalization for noise reduction. It also presented a comparative study between these proposed watermarking techniques and the existing state-of-the-art techniques. The evaluation metrics for the comparisons on standard 3DV streams include the stability, reliability, and robustness. The use of chaotic map interleaving with convolution coding and linear equalization minimized the ISI, which occurs in multi-path fading channels and increased the PSNR. Also, applying the chaotic map interleaving added some type of encryption and security to the compressed (DWT+SVD) watermarked 3DV data transmission through the wireless channel. Simulation results showed a good match between objective performance metrics and subjective fidelity measures (human visual perception). This study confirmed that using the chaotic map jointly with convolution coding and the linear equalization can be applied in the case of severe lossy wireless channel conditions such as the simulated channel that is influenced by the AWGN and Rayleigh fading, while achieving satisfactory objective and subjective results. Also, experimental results revealed the superiority of the proposed hybrid techniques in maintaining high robustness and fidelity in the presence of different multimedia attacks compared to the existing watermarking techniques. Moreover, the proposed techniques extract the watermark frames with high probability of detection and good 3DV perceptual quality.

\section{References}

1. Cagri O, Erhan E, Janko C, Ahmet K (2016) Adaptive delivery of immersive 3D multi-view video over the Internet. Journal of Multimedia Tools and Applications 75(20):12431-12461

2. Zhang Q, Wang X, Huang X, Su R, Gan Y (2015) Fast mode decision algorithm for 3D-HEVC encoding optimization based on depth information. Digital Signal Processing 44:37-46

3. Zhang Q, Chang H, Wu Q, Gan Y (2016) Fast motion and disparity estimation for HEVC based 3D video coding. Multidimensional Systems and Signal Processing 27(3):743-761

4. Zhou Y, Xiang W, Wang G (2015) Frame loss concealment for multiview video transmission over wireless multimedia sensor networks. IEEE Sensors Journal 15(3):1892-1901

5. Ramzan N, Amira A, Grecos C (2013) Efficient transmission of multiview video over unreliable channels. In Proceedings 2013 20th IEEE International Conference on Image Processing (ICIP) pp 1885-1889

6. Salim O, Wei X, Leis J (2013) An efficient unequal error protection scheme for 3-D video transmission. In Proceedings 2013 IEEE Wireless Communications and Networking Conference (WCNC) pp 4077-4082

7. Ibrahim A, Sadka A (2014) Error resilience and concealment for multiview video coding. In Proceedings 2014 IEEE International Symposium on Broadband Multimedia Systems and Broadcasting (BMSB) pp 1-5

8. Kuo C, Shih C, Hwang W (2012) Modeling and analysis of frame-level forward error correction for mpeg video over burst-loss channels. Appl. Math. Inf. Sci. 5(2S):16S-87S

9. Yongkai H, El-Hajjar M, Hanzo L (2013) Inter layer FEC aided unequal error protection for multilayer video transmission in mobile TV. IEEE Transactions on Circuits and Systems for Video Technology 23(9):1622-1634

10. Lei S, Lau V (2002) Performance analysis of adaptive interleaving for OFDM systems. IEEE Transactions On Vehicular Technology 51(3):435-444

11. El-Bakery E, El-Rabaie S, Zahran O, El-Samie F (2017) Chaotic Interleaving for the Transmission of Compressed Video Frames with Self-Embedded Digital Signatures. Wireless Personal Communications 96(2):1635-1651 
12. Al-kamali F, Dessouky M, Sallam B, El-Samie F (2008) Frequency domain interference cancellation for single carrier cyclic prefix CDMA systems. Progress In Electromagnetics Research B, PIERB 3:255-269

13. Al-kamali F, Dessouky M, Sallam B, El-Samie F (2009) Performance evaluation of cyclic prefix CDMA systems with frequency domain interference cancellation. Elsevier Inc Digital Signal Processing Journal 19(1):2-13

14. Campisi P (2008) Object-oriented stereo-image digital watermarking. Journal of electronic imaging 17(4):043024-043024

15. Niu Y, Souidene W, Beghdadi A (2011) A visual sensitivity model based stereo image watermarking scheme. In: IEEE European Workshop on Visual Information Processing (EUVIP) pp 211-215

16. Lin Y, Wu J (2012) Unseen visible watermarking for color plus depth map 3D images. In: IEEE International Conference on Acoustics, Speech and Signal Processing (ICASSP) pp 1801-1804

17. Lin Y, Wu J (2011) A digital blind watermarking for depth-image-based rendering 3D images. IEEE transactions on Broadcasting 57(2):602-611

18. Kim H, Lee J, Oh T, Lee H (2012) Robust DT-CWT watermarking for DIBR 3D images. IEEE Transactions on Broadcasting 58(4):533-543

19. Khalid A (2017) Utilization of watermarking schemes for securing digital images. Master Thesis, Department of Computer Engineering, Faculty of Electronic Engineering, Menoufia University

20. Wang S, Cui C, Niu X (2014) Watermarking for DIBR 3D images based on SIFT feature points. Measurement 48:54-62

21. Franco-Contreras J, Baudry S, Doërr G (2011) Virtual view invariant domain for 3D video blind watermarking. In: IEEE International Conference on Image Processing (ICIP) pp 2761-2764

22. Lee M, Lee J, Lee H (2011) Perceptual watermarking for 3D stereoscopic video using depth information. In: Seventh International Conference on Intelligent Information Hiding and Multimedia Signal Processing (IIH-MSP) pp 81-84

23. Swati S, Hayat K, Shahid Z (2014) A watermarking scheme for high efficiency video coding (HEVC). PloS one 9(8):e105613

24. Ogawa K, Ohtake G (2015) Watermarking for HEVC/H.265 stream. In: IEEE International Conference on Consumer Electronics (ICCE) pp 102-103

25. Zhang J, Ho A (2006) Efficient video authentication for H. 264/AVC. In: First International Conference on Innovative Computing, Information and Control (ICICIC) pp 46-49

26. Zhang J, Ho A, Qiu G, Marziliano P (2007) Robust video watermarking of H.264/AVC. IEEE Transactions on Circuits and Systems II: Express Briefs 54(2):205-209

27. Qiu G, Marziliano P, Ho A, He D, Sun Q (2004). A hybrid watermarking scheme for H.264/AVC video. In: IEEE International Conference on Pattern Recognition (ICPR) pp 865-868

28. Kuo T, Lo Y (2010) A hybrid scheme of robust and fragile watermarking for H. 264/AVC video. In: IEEE International Symposium on Broadband Multimedia Systems and Broadcasting (BMSB) pp $1-6$

29. Noorkami M, Mersereau R (2005) Compressed-domain video watermarking for H.264. In: IEEE International Conference on Image Processing (ICIP) pp II-890

30. Noorkami M, Mersereau R (2007) A framework for robust watermarking of H.264-encoded video with controllable detection. IEEE Transactions on information forensics and security 2(1):14-23

31. Noorkami M, Mersereau R (2008) Digital video watermarking in P-frames with controlled video bit-rate increase. IEEE transactions on information forensics and security 3(3):441-455

32. Esen E, Alatan A (2011) Robust video data hiding using forbidden zone data hiding and selective embedding. IEEE transactions on Circuits and Systems for Video Technology 21(8):1130-1138

33. Xu D, Wang R, Wang J (2011) A novel watermarking scheme for H.264/AVC video authentication. Signal Processing: Image Communication 26(6):267-279

34. Dutta T, Sur A, Nandi S (2013) A robust compressed domain video watermarking in P-frames with controlled bitrate increase. In: National Conference on Communications (NCC) pp 1-5

35. Stütz T, Autrusseau F, Uhl A (2014) Non-blind structure-preserving substitution watermarking of H.264/CAVLC inter-frames. IEEE Transactions on Multimedia 16(5):1337-1349

36. Su P, Wu C, Chen F, Wu C, Wu Y (2011) A practical design of digital video watermarking in $\mathrm{H}$. 264/AVC for content authentication. Signal Processing: Image Communication 26(8):413-426

37. T. Y. Chung, S. Sull, and C. S. Kim, " Frame loss concealment for stereoscopic video plus depth sequences”, IEEE Transactions on Consumer Electronics, 57(3), 1336-1344, 2011.

38. W. El-Shafai, B. Hrušovský, M. El-Khamy, and M. El-Sharkawy , “ Joint space-time-view error concealment algorithms for 3D multi-view video", In Image Processing (ICIP), 2011 18th IEEE International Conference on (pp. 2201-2204). IEEE., (2011, September) 
39. Schulze H, Luders C (2005) Theory and application of ofdm and cdma wideband wireless communication. John Wiley

40. El-Shafai W, El-Bakary E, El-Rabaie S, Zahran O, El-Halawany M, El-Samie F (2017). Efficient 3D Watermarked Video Communication with Chaotic Interleaving, Convolution Coding, and LMMSE Equalization. 3D Research 8(2):21

41. El-Bakary E, Hassan E, Zahran O, El-Dolil S, El-Samie F (2013). Efficient image transmission with multi-carrier CDMA. Wireless personal communications 69(2):979-994

42. Mueller K, Vetro A (2014) Common test conditions of 3D-MVV core experiments. Joint Collaborative Team on 3D Video Coding Extensions JCT3V-G1100, 7th Meeting: San Jose, USA

43. Conti A, Dardari D, Paolini G, Andrisano O (2003) Bluetooth and IEEE 802.11b coexistence: analytical performance evaluation in fading channels. IEEE Journal on Selected Areas in Communications 21(2):259-269

44. Rajab L, Al-Khatib T, Al-Haj A (2009) Video watermarking algorithms using the SVD transform. European Journal of Scientific Research 30(3):389-401

45. Khalid A, El-Sayed M, Fathi E, Faragallah O, ELmhalawy A, Shehata A (2016) A comparative study for color systems used in the DCT-DWT watermarking algorithm. Advances in Science, Technology and Engineering Systems Journal 1(5):42-49 
Figures

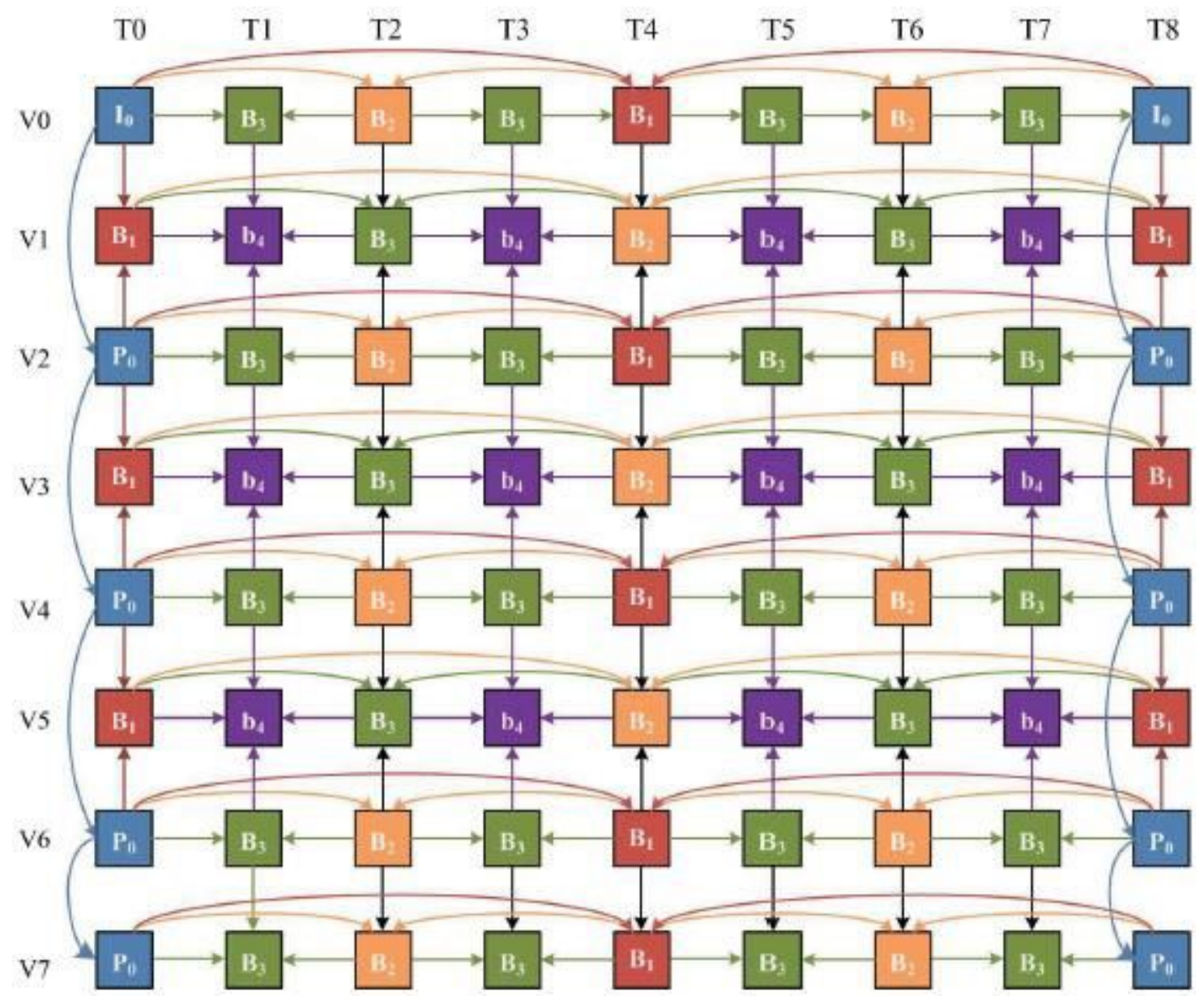

Figure 1

3D video prediction coding model.

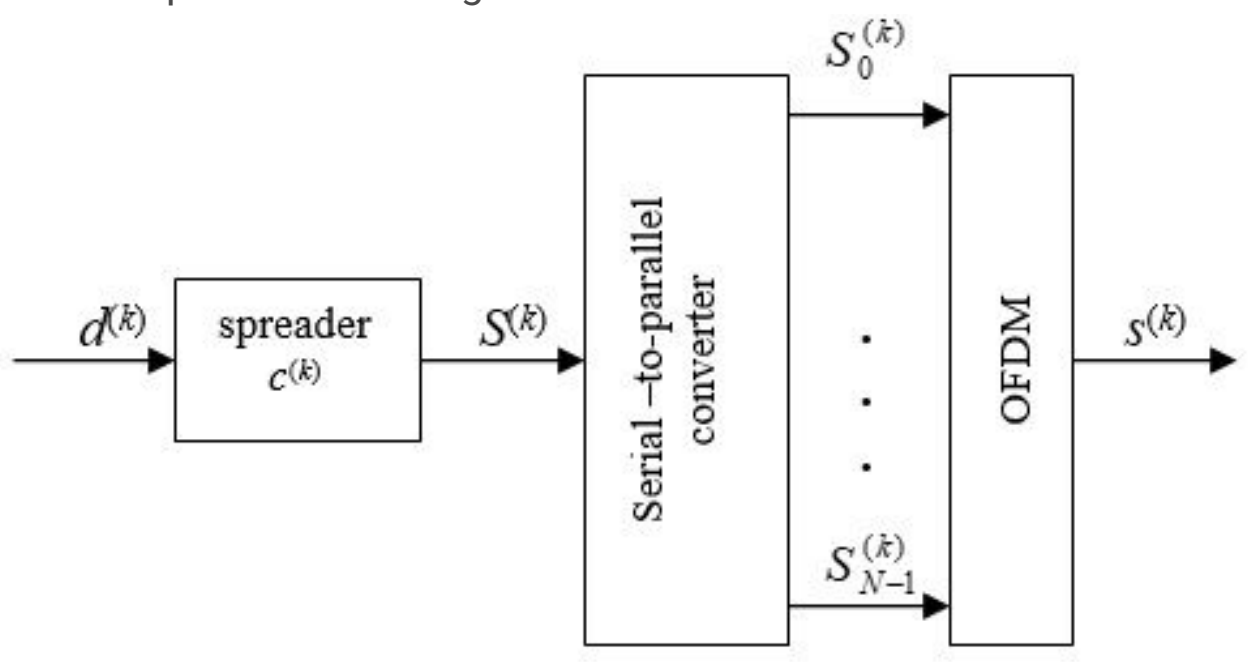

Figure 2

Multi-carrier spread spectrum signal generation. 


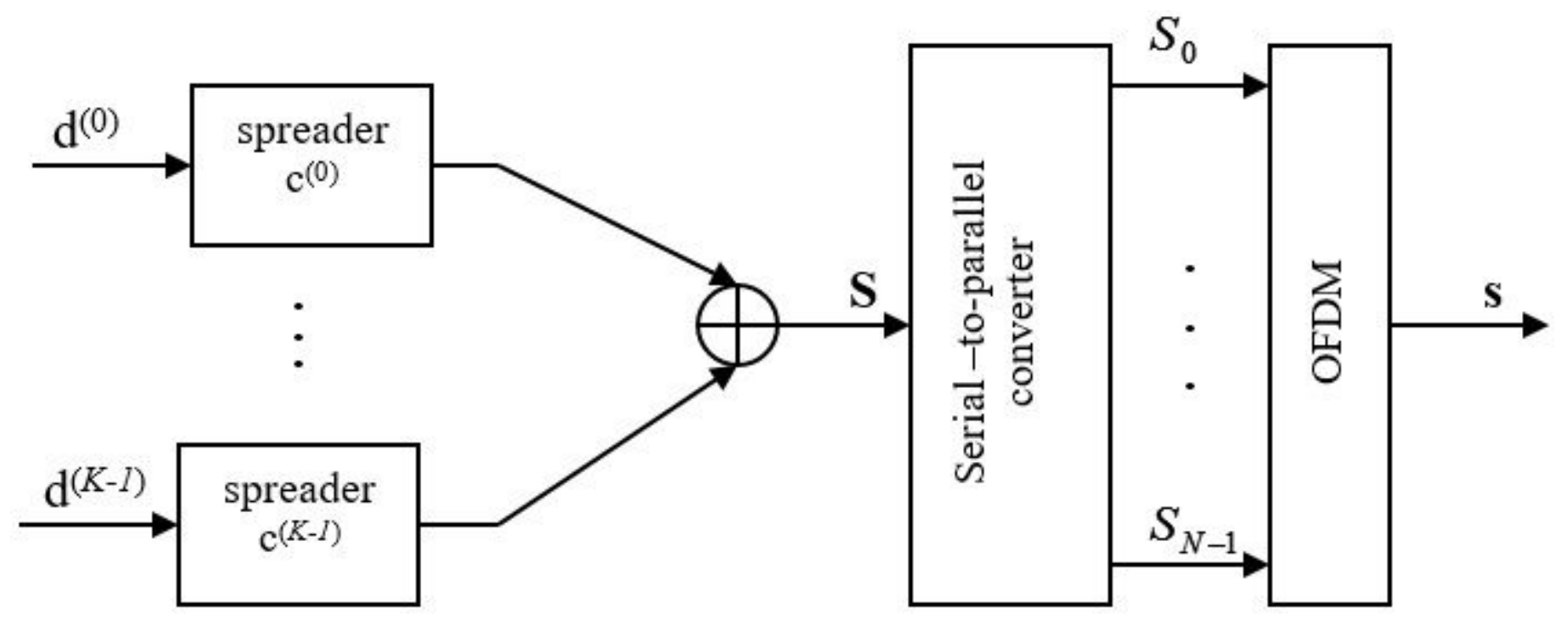

Figure 3

MC-CDMA transmitter.

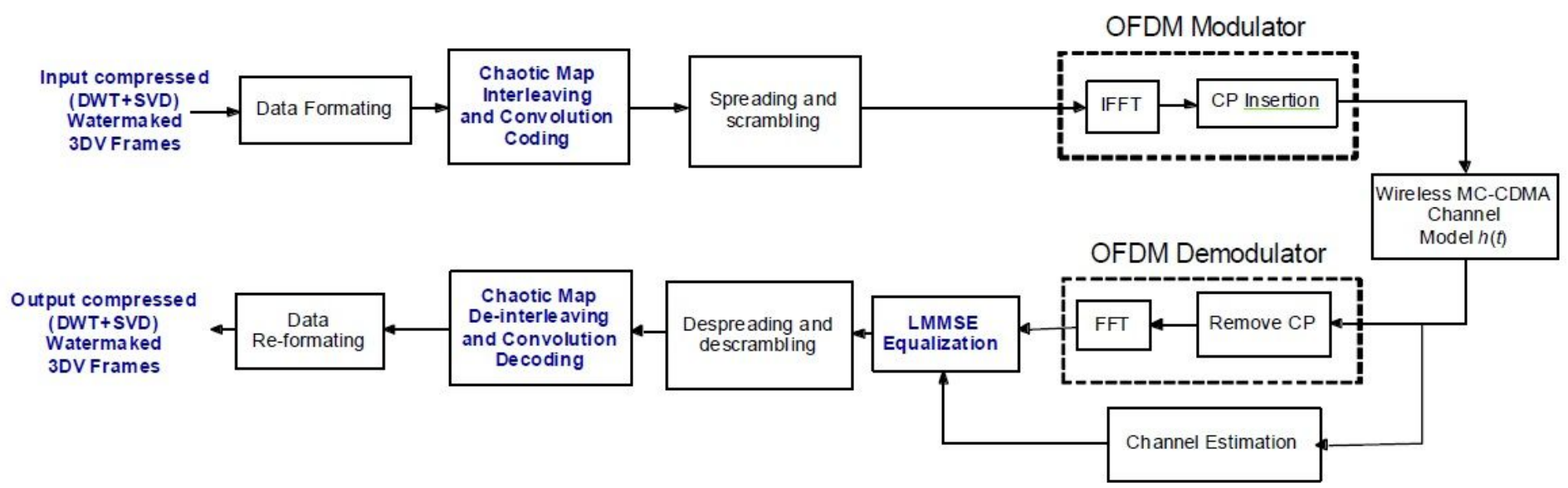

\section{Figure 4}

Block diagram of the proposed joint framework for compressed (DWT+SVD) watermarked 3DV transmission over wireless MC-CDMA system. 


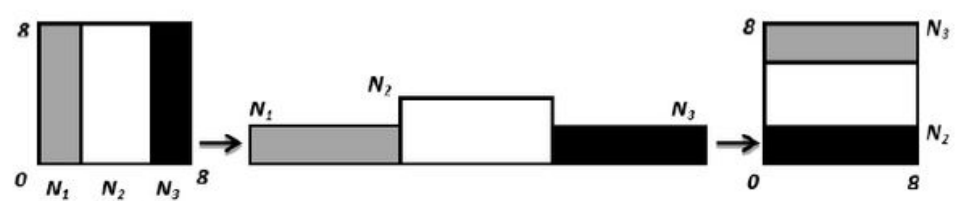

(a)

$$
N_{1}=2 \quad N_{2}=4 \quad N_{3}=2
$$

\begin{tabular}{|cc|cccc|cc|}
\hline$Y_{1}$ & $Y_{2}$ & $Y_{3}$ & $Y_{4}$ & $Y_{5}$ & $Y_{6}$ & $Y_{7}$ & $Y_{8}$ \\
$Y_{9}$ & $Y_{10}$ & $Y_{11}$ & $Y_{12}$ & $Y_{13}$ & $Y_{14}$ & $Y_{15}$ & $Y_{16}$ \\
\cline { 3 - 5 }$Y_{17}$ & $Y_{18}$ & $Y_{19}$ & $Y_{20}$ & $Y_{21}$ & $Y_{22}$ & $Y_{23}$ & $Y_{24}$ \\
$Y_{25}$ & $Y_{26}$ & $Y_{27}$ & $Y_{28}$ & $Y_{29}$ & $Y_{30}$ & $Y_{31}$ & $Y_{32}$ \\
\hline$Y_{33}$ & $Y_{34}$ & $Y_{35}$ & $Y_{36}$ & $Y_{37}$ & $Y_{38}$ & $Y_{39}$ & $Y_{40}$ \\
$Y_{41}$ & $Y_{42}$ & $Y_{43}$ & $Y_{44}$ & $Y_{45}$ & $Y_{46}$ & $Y_{47}$ & $Y_{48}$ \\
\cline { 3 - 4 }$Y_{49}$ & $Y_{50}$ & $Y_{51}$ & $Y_{52}$ & $Y_{53}$ & $Y_{54}$ & $Y_{55}$ & $Y_{56}$ \\
$Y_{57}$ & $Y_{58}$ & $Y_{59}$ & $Y_{60}$ & $Y_{61}$ & $Y_{62}$ & $Y_{63}$ & $Y_{64}$ \\
\hline
\end{tabular}

PM Output

\begin{tabular}{|lccccccc|}
\hline$Y_{31}$ & $Y_{23}$ & $Y_{15}$ & $Y_{7}$ & $Y_{32}$ & $Y_{24}$ & $Y_{16}$ & $Y_{8}$ \\
\hline$Y_{63}$ & $Y_{55}$ & $Y_{47}$ & $Y_{39}$ & $Y_{64}$ & $Y_{56}$ & $Y_{48}$ & $Y_{40}$ \\
\hline$Y_{11}$ & $Y_{3}$ & $Y_{12}$ & $Y_{4}$ & $Y_{13}$ & $Y_{5}$ & $Y_{14}$ & $Y_{6}$ \\
\hline$Y_{27}$ & $Y_{19}$ & $Y_{28}$ & $Y_{20}$ & $Y_{29}$ & $Y_{21}$ & $Y_{30}$ & $Y_{22}$ \\
\hline$Y_{43}$ & $Y_{35}$ & $Y_{44}$ & $Y_{36}$ & $Y_{45}$ & $Y_{37}$ & $Y_{46}$ & $Y_{38}$ \\
\hline$Y_{59}$ & $Y_{51}$ & $Y_{60}$ & $Y_{52}$ & $Y_{61}$ & $Y_{53}$ & $Y_{32}$ & $Y_{54}$ \\
\hline$Y_{25}$ & $Y_{17}$ & $Y_{9}$ & $Y_{1}$ & $Y_{26}$ & $Y_{18}$ & $Y_{10}$ & $Y_{2}$ \\
\hline$Y_{57}$ & $Y_{49}$ & $Y_{41}$ & $Y_{33}$ & $Y_{58}$ & $Y_{50}$ & $Y_{42}$ & $Y_{34}$ \\
\hline
\end{tabular}

CMR Output

(b)

Figure 5

Chaotic interleaving example using a secret key $=(2,4,2)$ : (a) Discretize Baker map, (b) An $8 \times 8$ randomizations output.

Figure 6

A proposed convolution encoder, where $x(i)$ is an input information bit stream and $c(i)$ is an output encoded bit stream. 


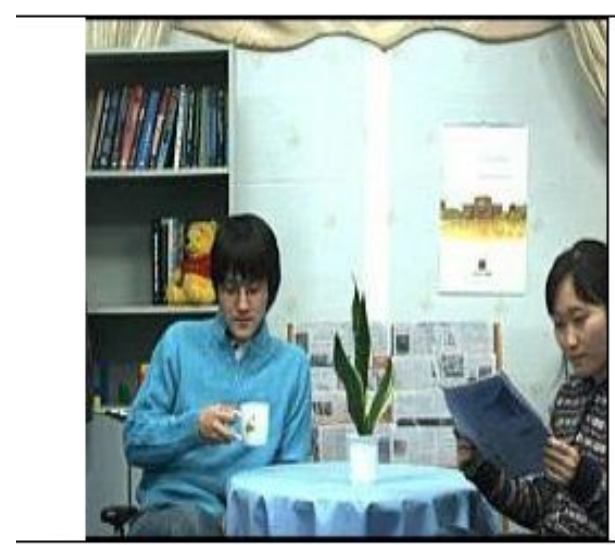

(a) Original 3DV Newspaper host frame 5 .

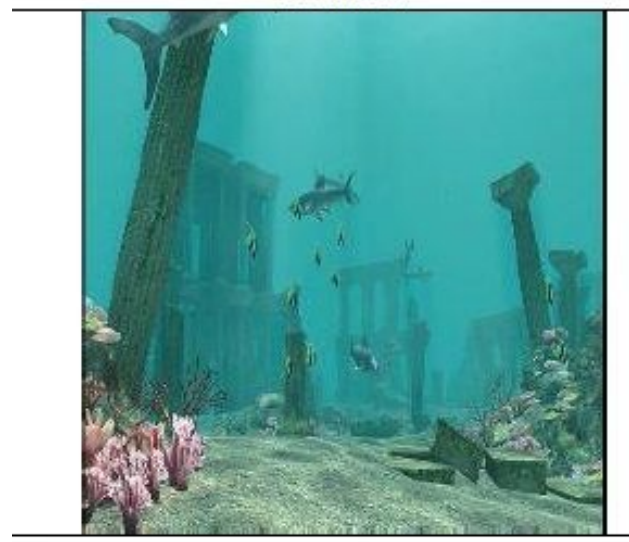

(d) Original 3DV Shark host frame 5.

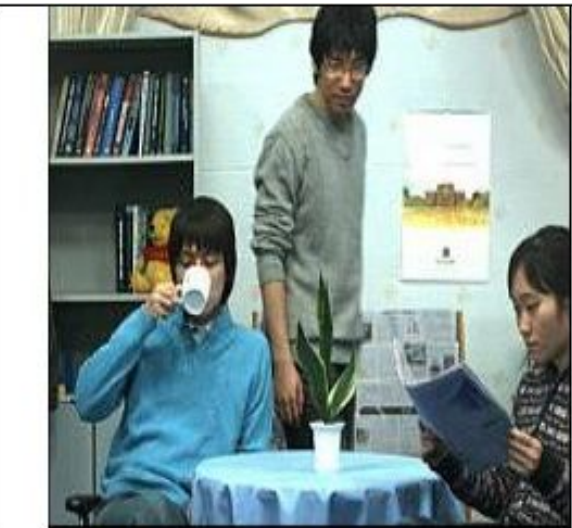

(b) Original 3DV Newspaper watermark frame 70 .

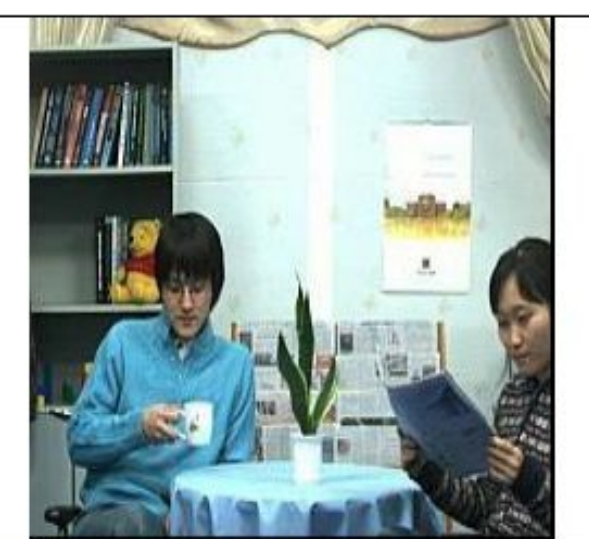

(c) Watermarked 3DV Newspaper with PSNR $=46.5652$.

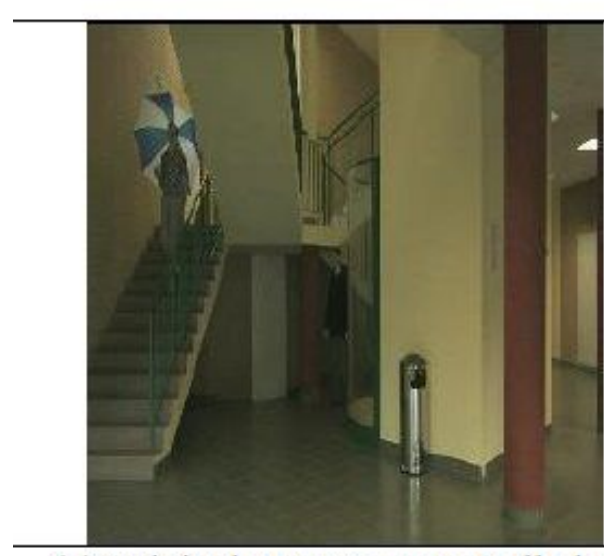

(g) Original 3DV Poznan_Hall2 host frame 50 . (e) Original 3DV Shark watermark frame 250 .

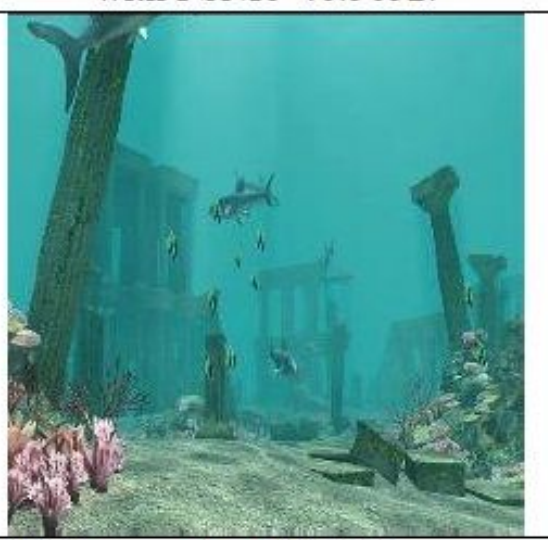

(f) Watermarked 3DV Newspaper with PSNR=47.7233.

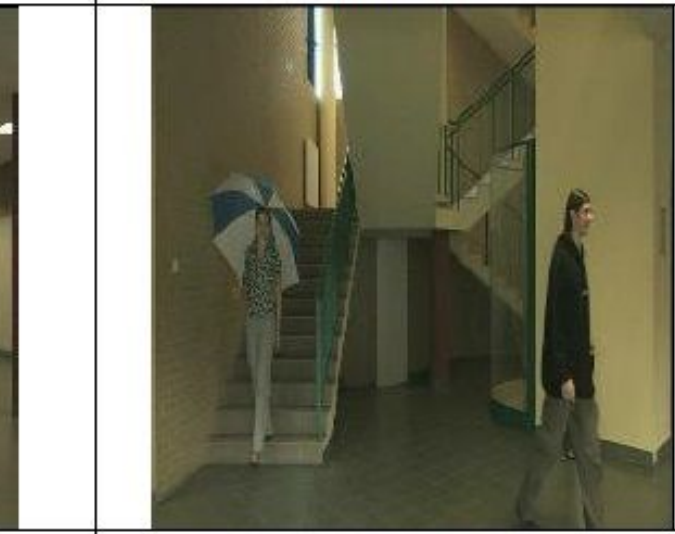

(h) Original 3DV Poznan_Hall2 watermark frame $20 \overline{0}$.

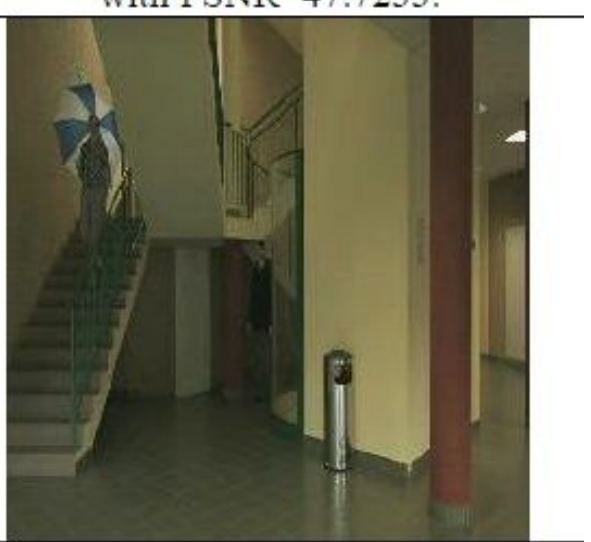

(i) Watermarked 3DV Newspaper with PSNR=64.1419.

\section{Figure 7}

3DV Newspaper, Shark, Poznan_Hall2 host, watermark, and watermarked frames in the case of error free channel. 


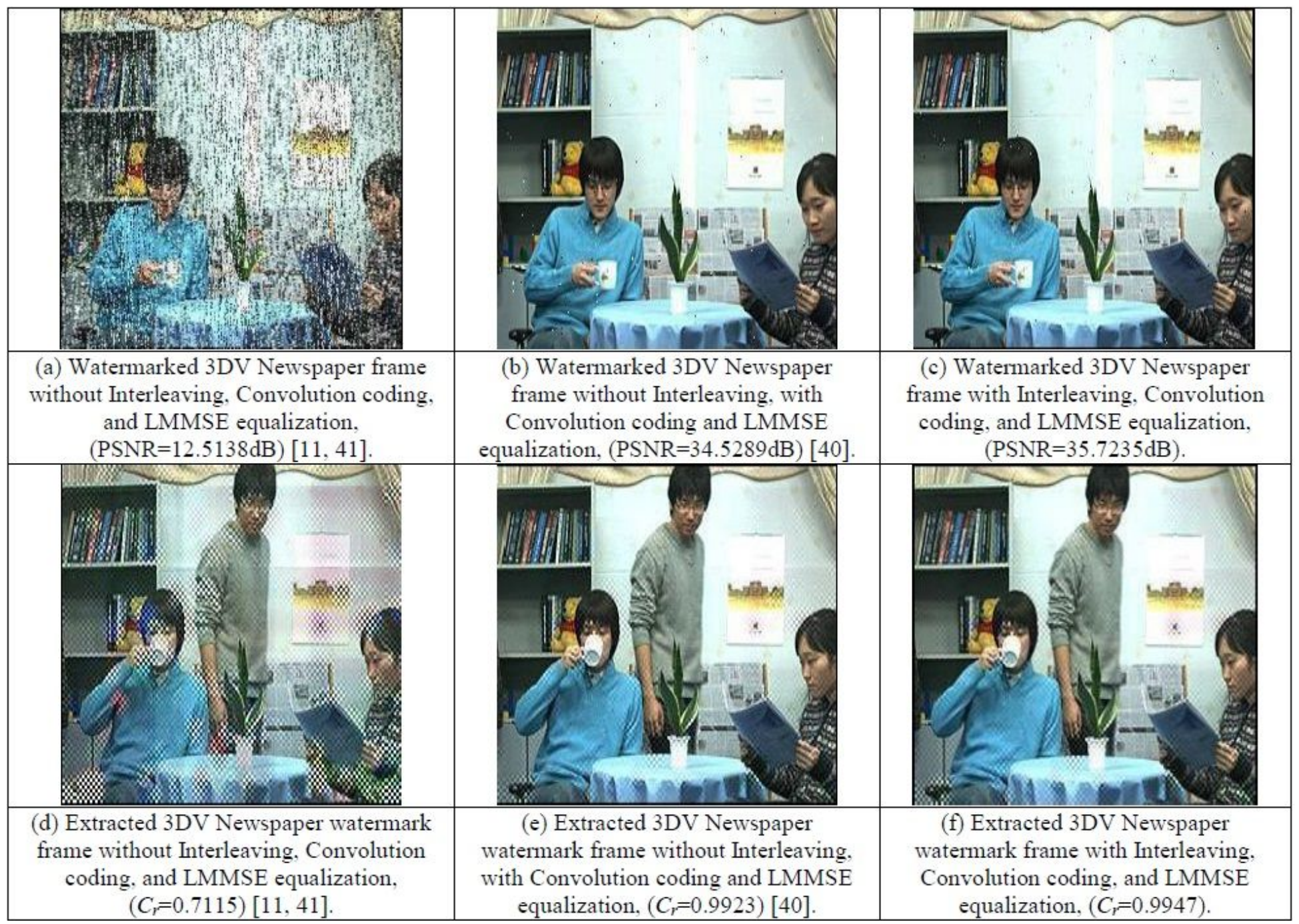

\section{Figure 8}

The received (DWT+SVD) watermarked 3DV Newspaper frame 5 and their extracted frames in the case of using the SUI-3 Raleigh fading channel influenced by AWGN 


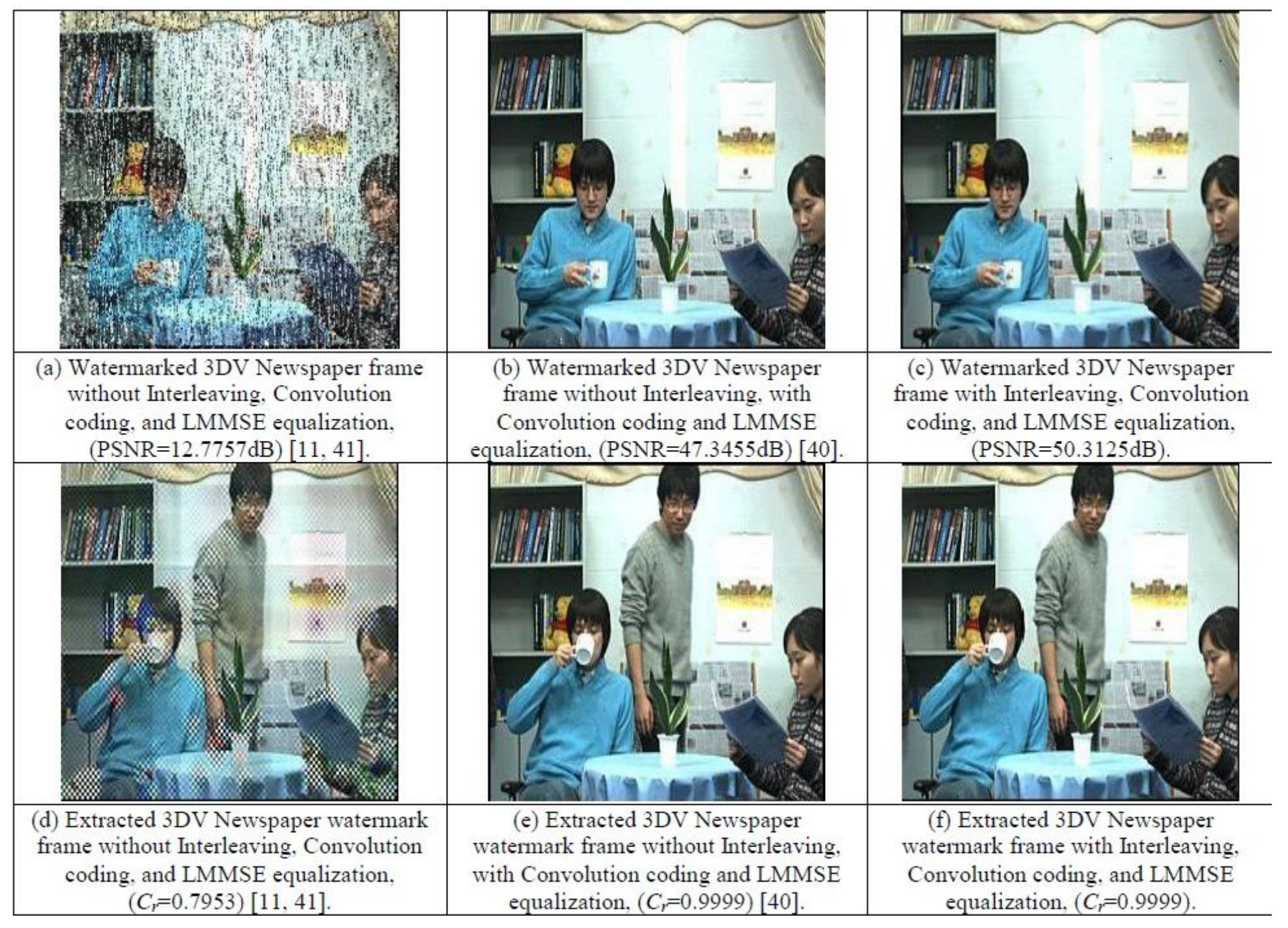

\section{Figure 9}

The received (DWT+SVD) watermarked 3DV Newspaper frame 5 and their extracted frames in the case of using the SUI-3 Raleigh fading channel influenced by AWGN at SNR=15dB. 


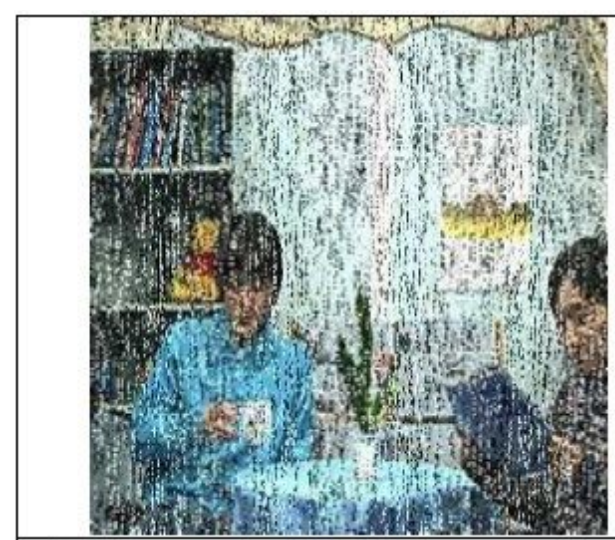

(a) Watermarked 3DV Newspaper frame without Interleaving, Convolution coding, and LMMSE equalization, $(\mathrm{PSNR}=12.8567 \mathrm{~dB})[11,41]$.

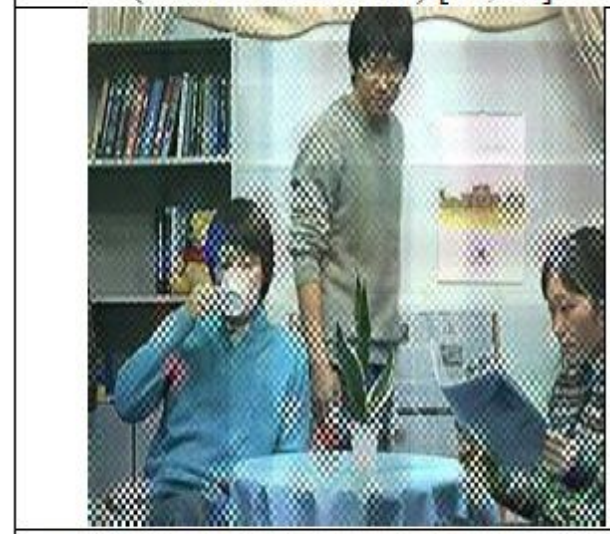

(d) Extracted 3DV Newspaper watermark frame without Interleaving, Convolution coding, and LMMSE equalization, $\left(C_{r}=0.8469\right)[11,41]$.

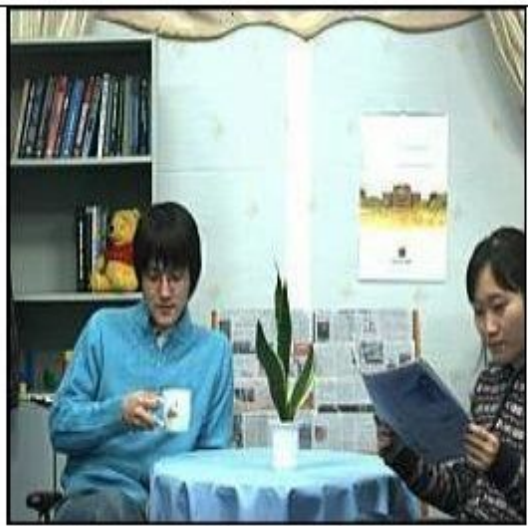

(b) Watermarked 3DV Newspaper frame without Interleaving, with Convolution coding and LMMSE equalization, $(\mathrm{PSNR}=54.1503 \mathrm{~dB})$ [40].

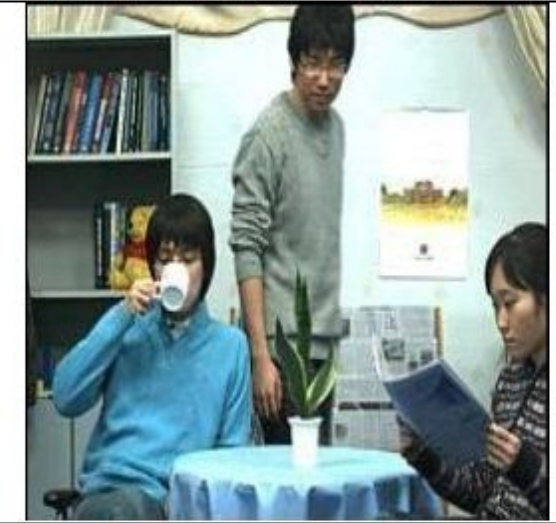

(e) Extracted 3DV Newspaper watermark frame without Interleaving, with Convolution coding and LMMSE equalization, $\left(C_{r}=0.9999\right)$ [40].

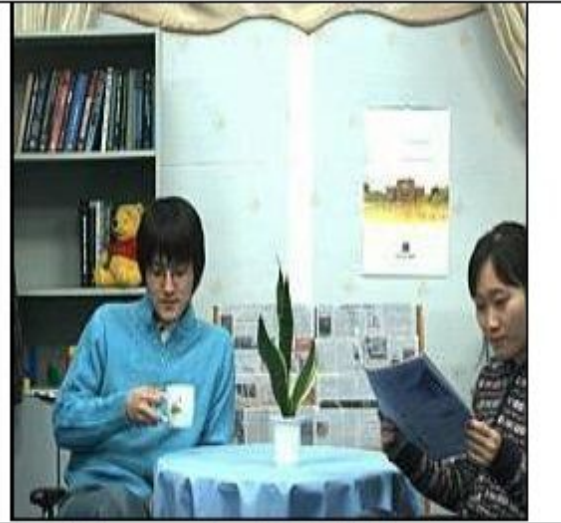

(c) Watermarked 3DV Newspaper frame with Interleaving, Convolution coding, and LMMSE equalization, $(\mathrm{PSNR}=60.1720 \mathrm{~dB})$.

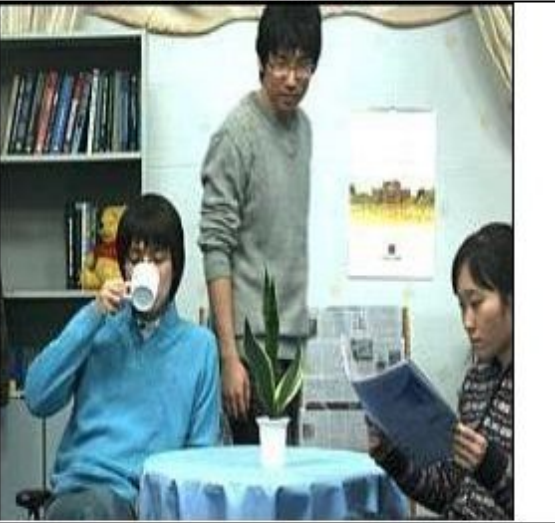

(f) Extracted 3DV Newspaper watermark frame with Interleaving, Convolution coding, and LMMSE equalization, $\left(C_{r}=0.9999\right)$.

\section{Figure 10}

The received (DWT+SVD) watermarked 3DV Newspaper frame 5 and their extracted frames in the case of using the SUI-3 Raleigh fading channel influenced by AWGN at SNR=20dB. 


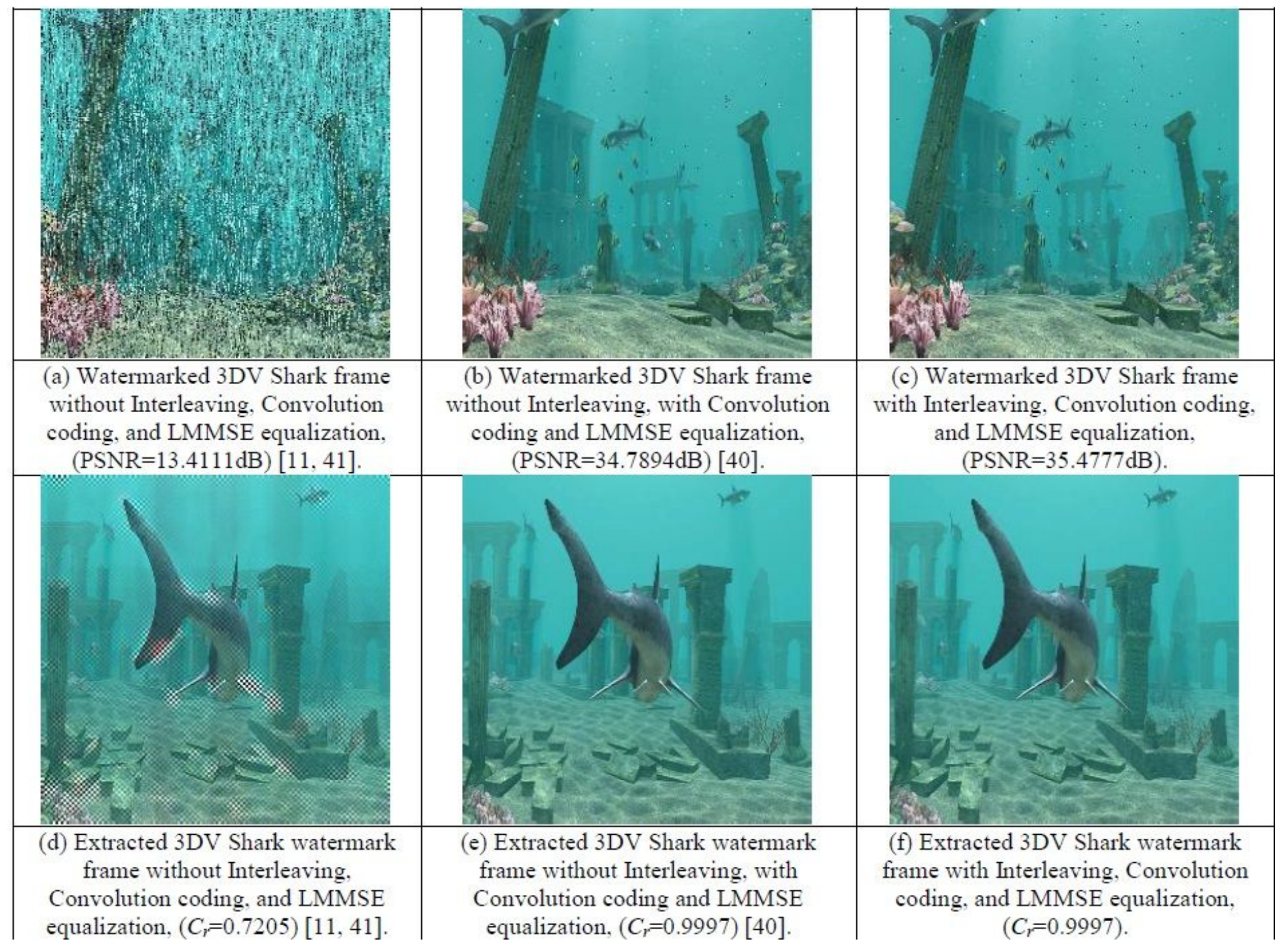

\section{Figure 11}

The received (DWT+SVD) watermarked 3DV Shark frame 5 and their extracted frames in the case of using the SUI-3 Raleigh fading channel influenced by AWGN at SNR=10dB./ 


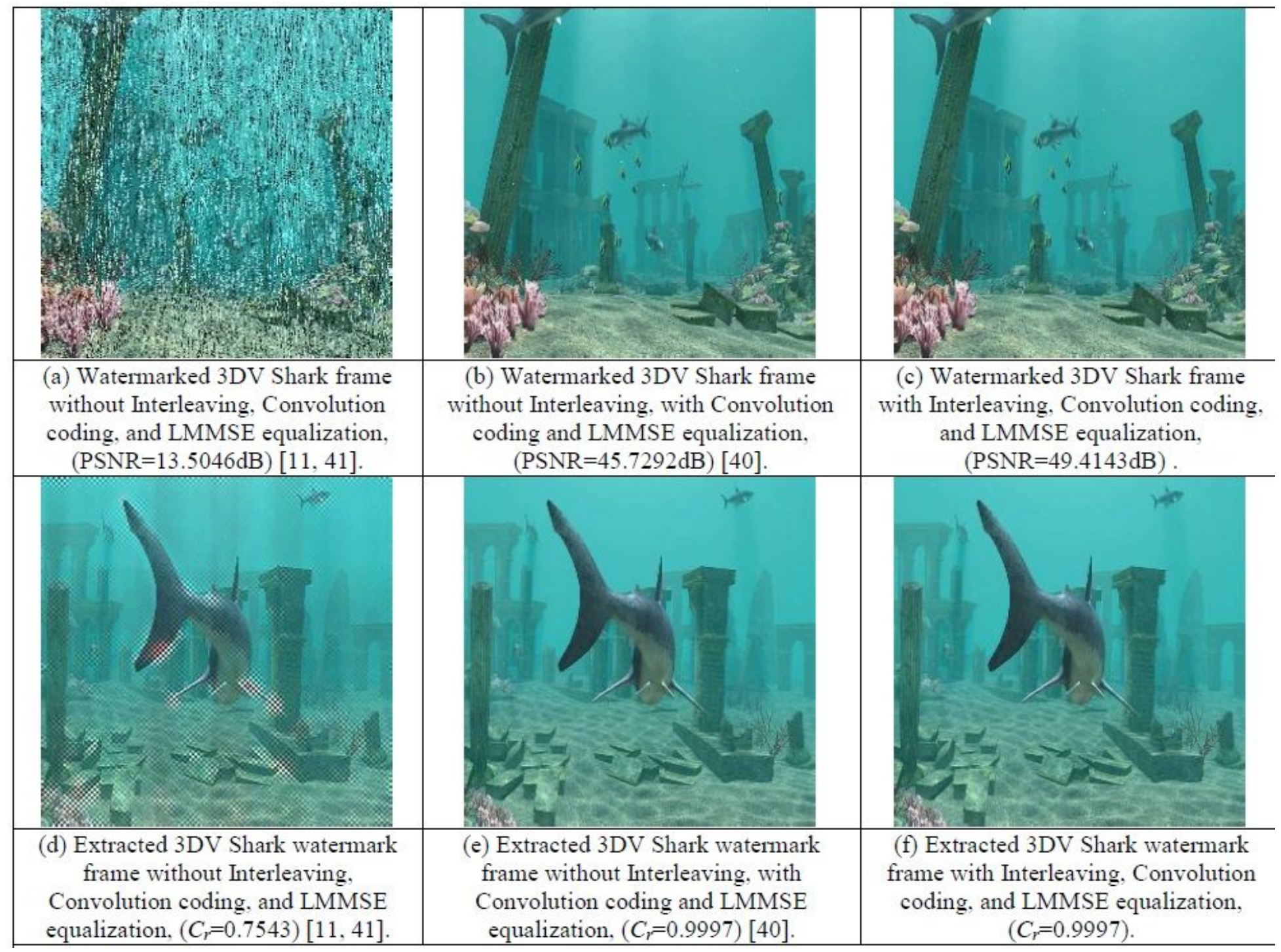

Figure 12

The received (DWT+SVD) watermarked 3DV Shark frame 5 and their extracted frames in the case of using the SUI-3 Raleigh fading channel influenced by AWGN at $S N R=15 \mathrm{~dB}$. 


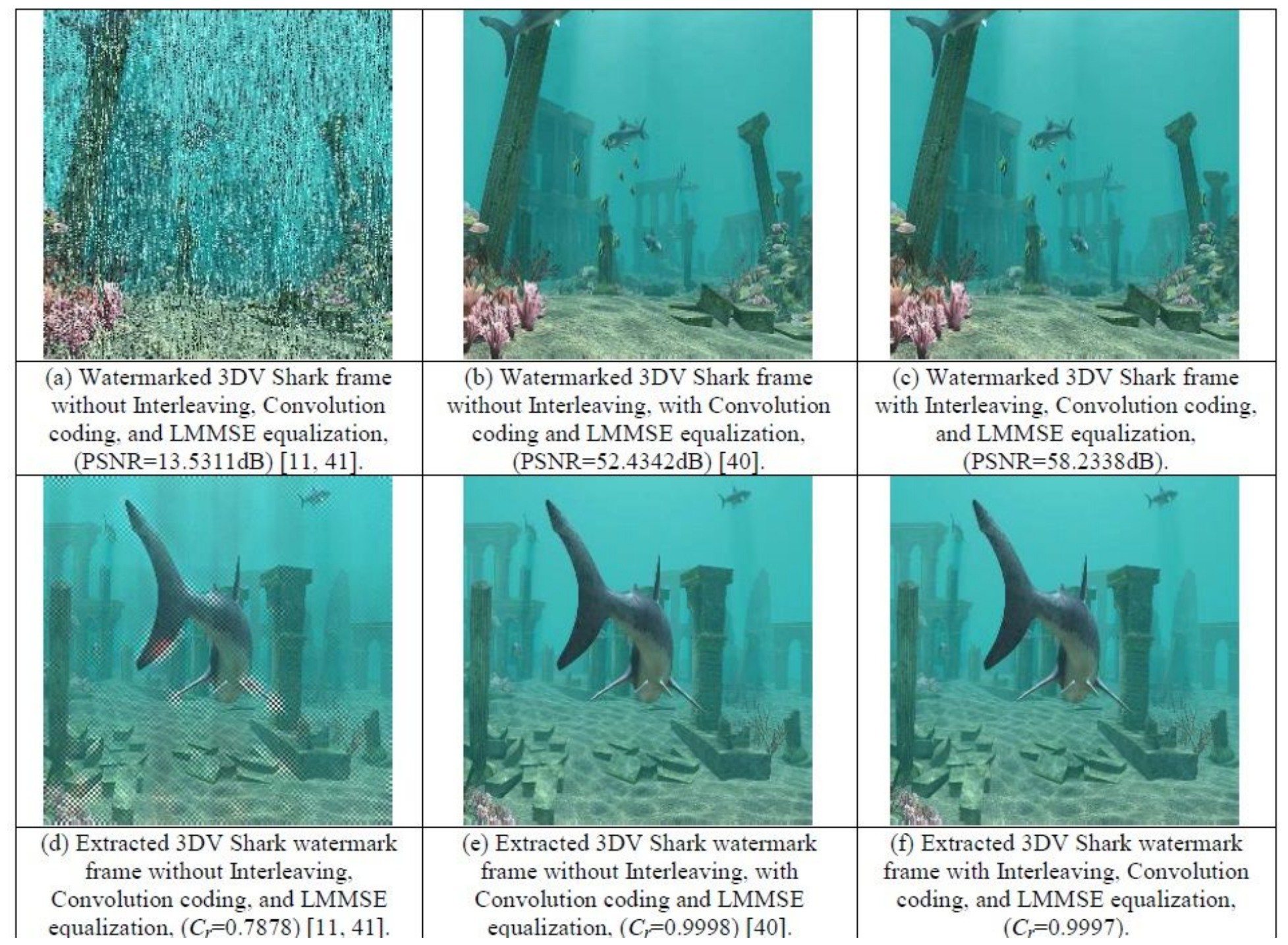

\section{Figure 13}

The received (DWT+SVD) watermarked 3DV Shark frame 5 and their extracted frames in the case of using the SUI-3 Raleigh fading channel influenced by AWGN at SNR=20dB. 


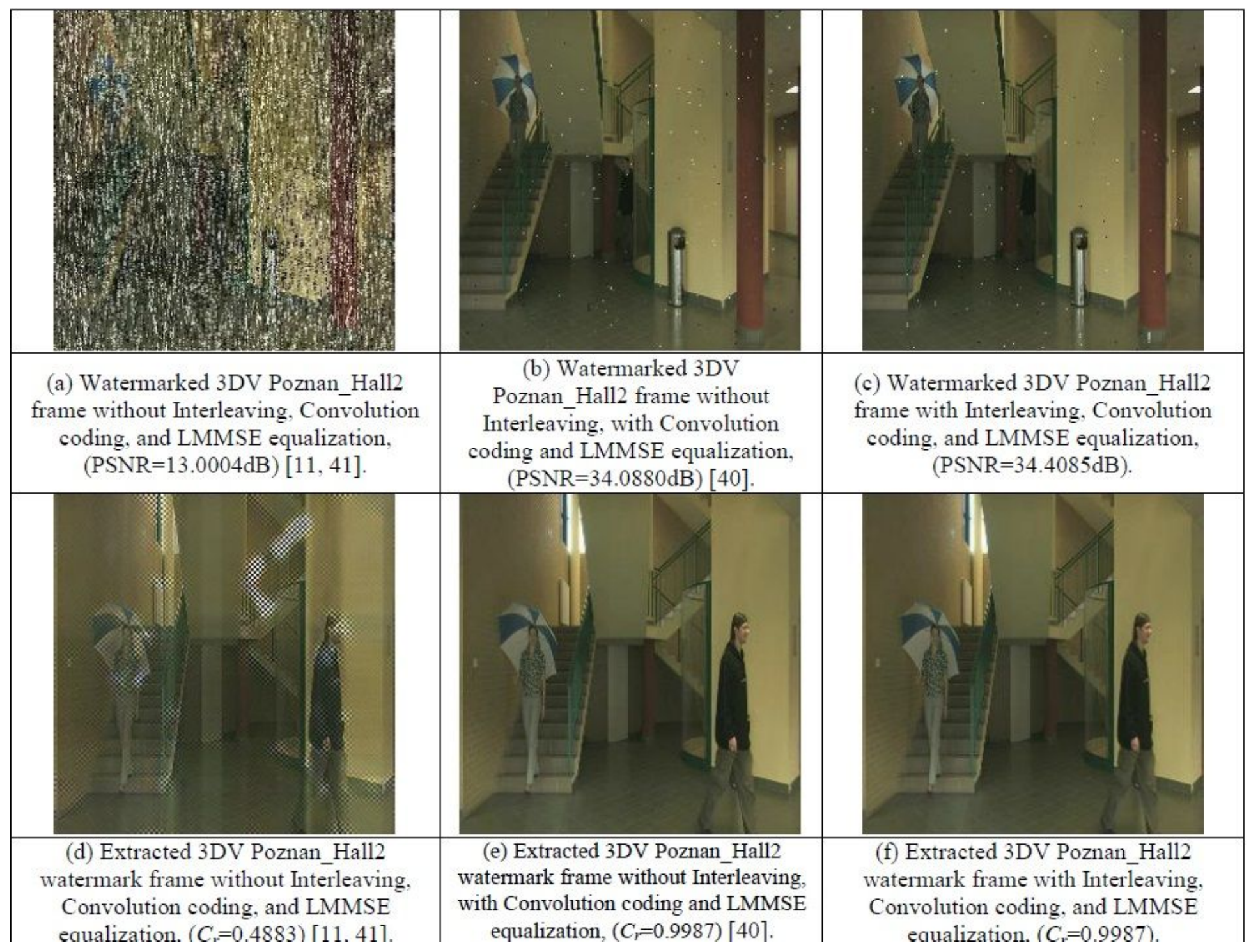

\section{Figure 14}

The received (DWT+SVD) watermarked 3DV Poznan_Hall2 frame 50 and their extracted frames in the case of using the SUI-3 Raleigh fading channel influenced by AWGN at SNR=10dB. 


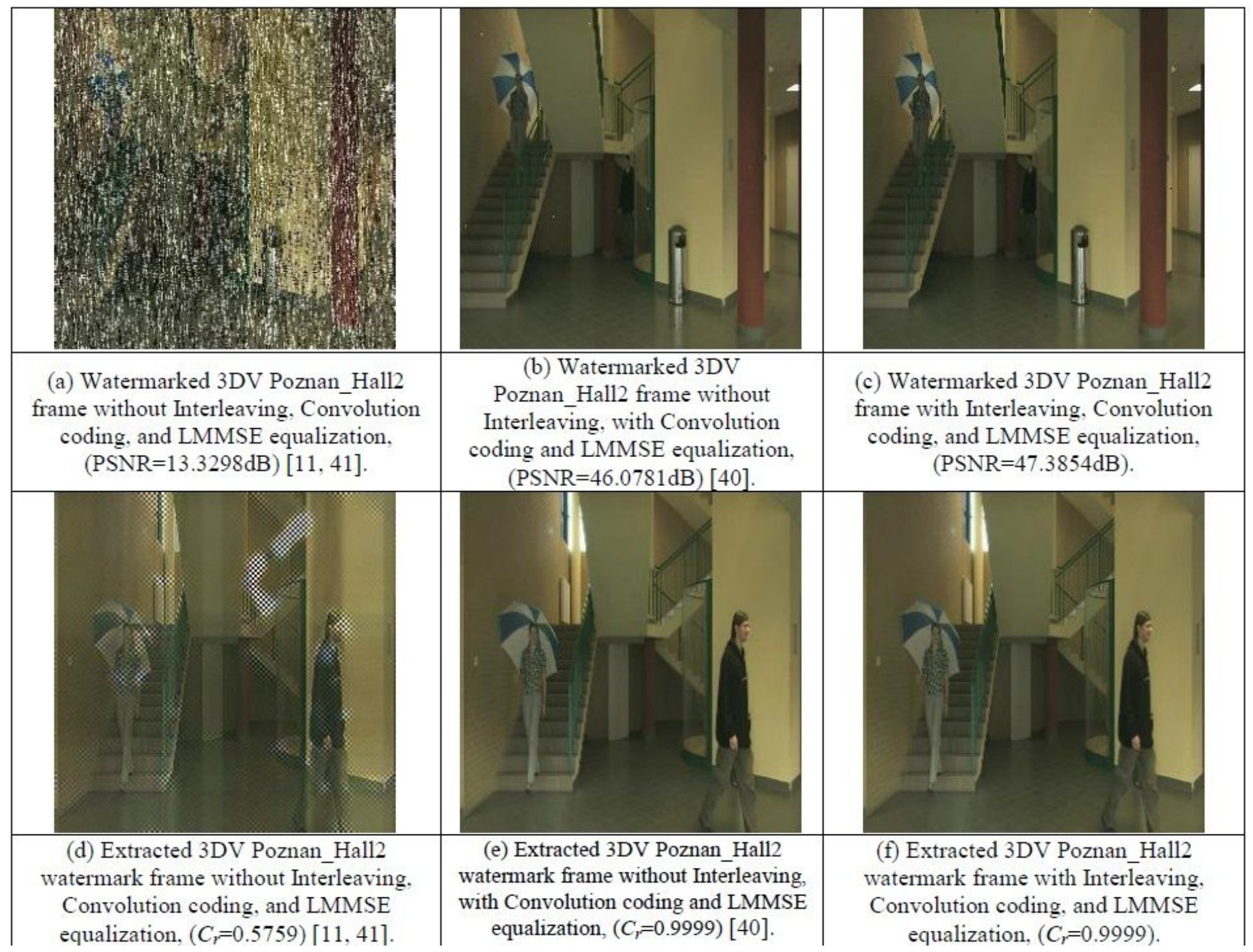

\section{Figure 15}

The received (DWT+SVD) watermarked 3DV Poznan_Hall2 frame 50 and their extracted frames in the case of using the SUI-3 Raleigh fading channel influenced by AWGN at SNR=15dB. 


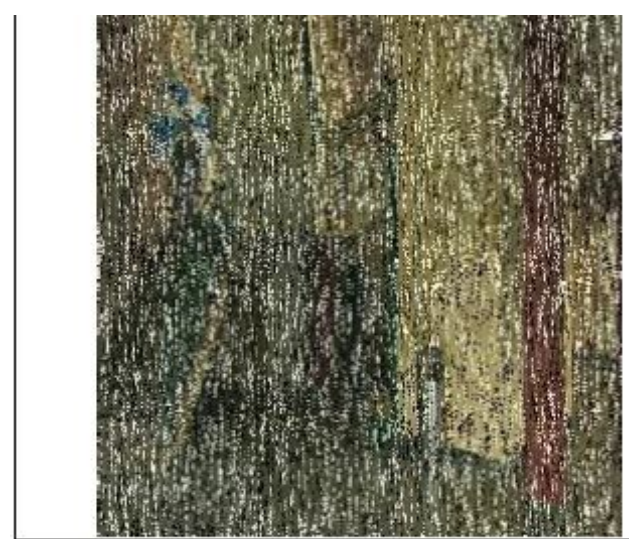

(a) Watermarked 3DV Poznan_Hall2 frame without Interleaving, Convolution coding, and LMMSE equalization, $(\mathrm{PSNR}=13.3298 \mathrm{~dB})[11,41]$.

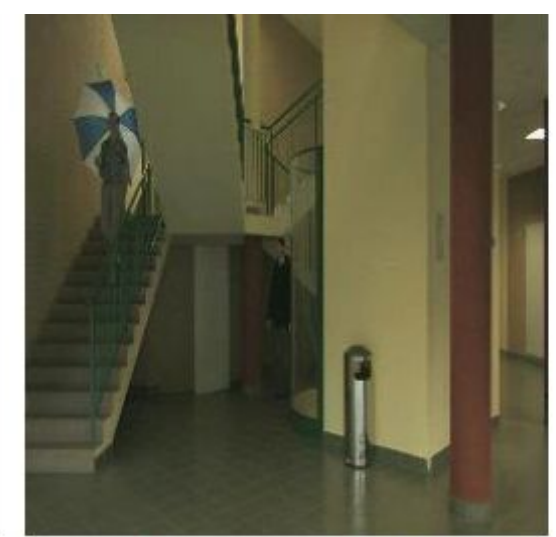

(b) Watermarked 3DV

Poznan_Hall2 frame without

Interleaving, with Convolution coding and LMMSE equalization, $(\mathrm{PSNR}=55.9293 \mathrm{~dB})[40]$.

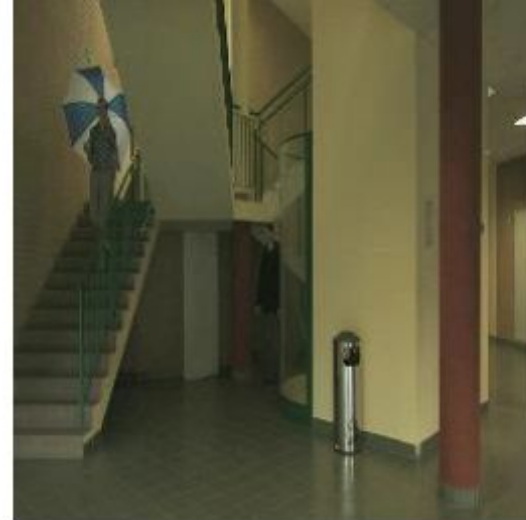

(c) Watermarked 3DV Poznan_Hall2 frame with Interleaving, Convolution coding, and LMMSE equalization, $(\mathrm{PSNR}=57.2132 \mathrm{~dB})$.

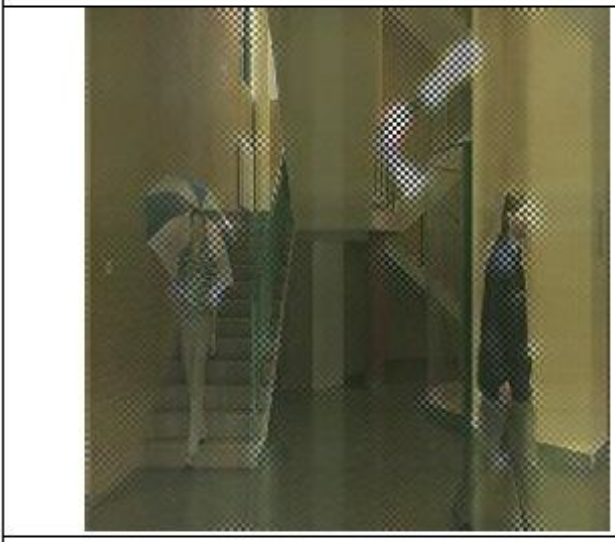

(d) Extracted 3DV Poznan_Hall2 watermark frame without Interleaving, Convolution coding, and LMMSE equalization, $\left(C_{r}=0.5770\right)[11,41]$.

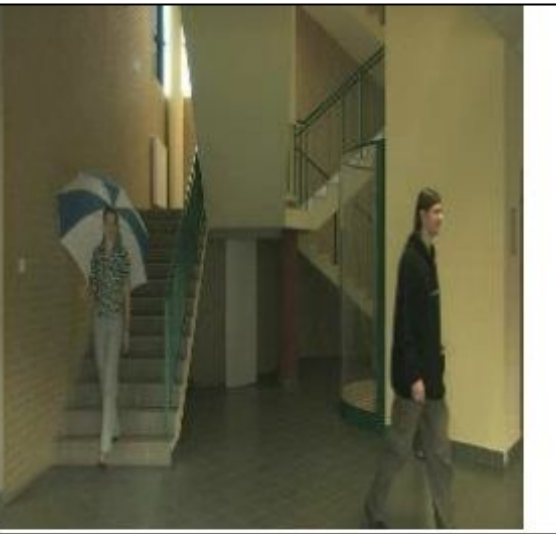

(f) Extracted 3DV Poznan_Hall2 watermark frame with Interleaving, Convolution coding, and LMMSE equalization, $\left(C_{r}=0.9999\right)$.

\section{Figure 16}

The received (DWT+SVD) watermarked 3DV Poznan_Hall2 frame 50 and their extracted frames in the case of using the SUI-3 Raleigh fading channel influenced by AWGN at SNR=20dB. 